$$
\begin{aligned}
& \text { IHES H1 } 93-63 \\
& \text { SW } 9411
\end{aligned}
$$

\title{
ON THE CAUCHY AND INVARIANT MEASURE PROBLEM FOR THE PERIODIC ZAKHAROV SYSTEM
}

\author{
Jean BOURGAIN
}
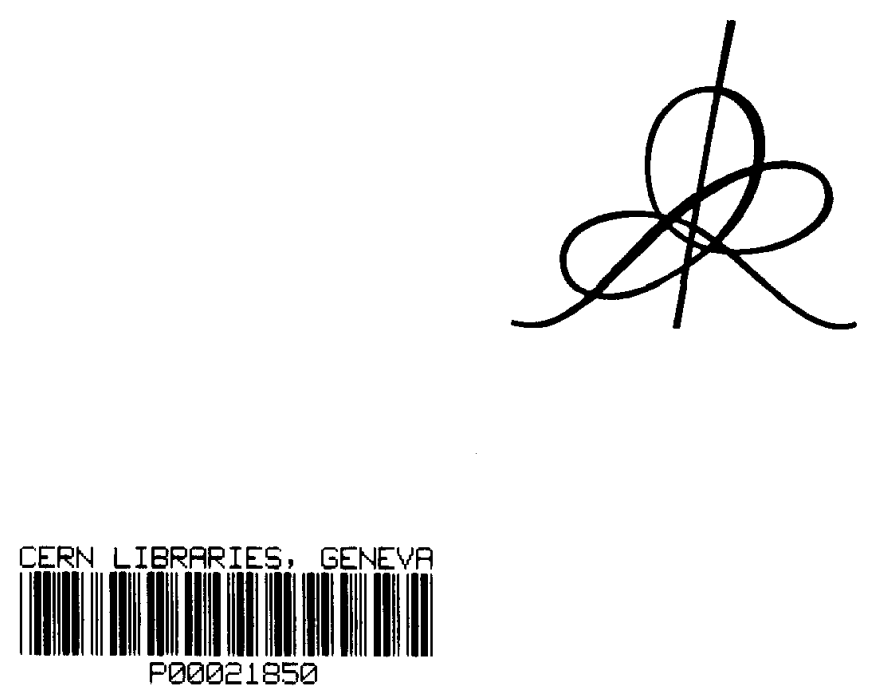

Institut des Hautes Etudes Scientifiques 35 , route de Chartres

91440 - Bures-sur-Yvette (France)

Novembre 1993

IHES $/ \mathrm{M} / 93 / 63$ 


$$
\text { L }
$$




\title{
ON THE CAUCHY AND INVARIANT MEASURE PROBLEM \\ FOR THE PERIODIC ZAKHAROV SYSTEM
}

\author{
Jean BOURGAIN $(*)$
}

\section{Introduction}

The purpose of this preliminary paper is to prove local and global existence and regularity theorems for the 1-dimensional Zakharov Model

$$
\left\{\begin{array}{l}
i u_{t}=-u_{x x}+n u \\
n_{t t}-n_{x x}=\left(|u|^{2}\right)_{x x} \\
u(x, 0)=\varphi, n(x, 0)=a, \partial_{t} n(x, 0)=b
\end{array}\right.
$$

in the space periodic case. Apparently no results were known so far on this problem, if one considers the periodic setting. Equations (1) are suspected to be nonintegrable, contrary to the NLSE $i u_{t}+u_{x x}+u|u|^{2}=0$. The technique used here is a Fourier analysis approach in the same spirit as earlier works in $\left[B_{1}, B_{2}\right]$ on NLSE and KdV type equations. In particular we prove following local wellposedness theorem.

Theorem 1. There are Sobolev exponents $0<\sigma<s<\frac{1}{2}<s_{1}<1$ such that (1) is locally wellposed for data $(u, a, b)$ satisfying

$$
\begin{gathered}
\varphi \in H^{s}(\mathrm{~T}), \sup _{k}|k|^{s_{1}}|\widehat{\varphi}(k)|<\infty \\
\sup _{k}|k|^{-\sigma}|\widehat{a}(k)|<\infty \text { and } \sup _{k}|k|^{-\sigma-1}|\widehat{b}(k)|<\infty .
\end{gathered}
$$

One shoud think here of $\sigma$ close to $0, s$ close to $\frac{1}{2}$. In particular, for $\left(H^{1}, L^{2}, H^{-1}\right)$-data, there is a global result

Theorem 2. The system (1) is globally wellposed for data $\varphi \in H^{1}, a \in L^{2}, b \in H^{-1}$.

due to the conservation of the Hamiltonian

$$
H_{Z}=\frac{1}{2} \int_{\mathbf{T}}\left[\left|u_{x}\right|^{2}+\frac{1}{2}\left(n^{2}+V^{2}\right)+n|u|^{2}\right] d x
$$

and the $L^{2}$-norm $\int_{\mathbf{T}}|u|^{2} d x$. Here $V$ is defined by

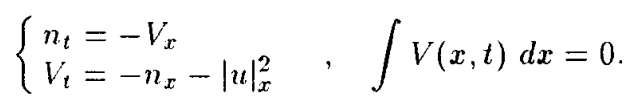

We believe that our method should be applicable in higher dimension too and plan to investigate this subsequently. There also should be a nonperiodic analogue to Theorem 2 for $H^{1}(\mathbf{R}), L^{2}(\mathbf{R}), H^{-1}(\mathbf{R})$ data, which would improve on the $H^{2}, H^{1}, L^{2}$ result of Ozawa and Tsutsumi ([O-T]).

(*) I.H.E.S., 35 route de Chartres, 91440 Bures-sur-yvette, FRANCE 
The stronger formulation of the local result in Theorem 1 is meant to cover the typical data in the support of the Gibbs measure, formally written before normalization as (see [L-R-S])

$$
e^{-\beta H_{Z}} \chi_{\left\{\int|u|^{2} d x \leq B\right\}} \prod_{x}\left[d^{2} u(x) d \tilde{n}(x) d \tilde{V}(x)\right]
$$

where $(\operatorname{Re} u, \operatorname{Im} u)$ and $(\tilde{n}, \tilde{V})$, with $\tilde{n}=2^{-1 / 2} n$ and $\widetilde{V}=2^{-1 / 2} D_{x}^{-1} V$, are pairs of conjugate variables. Here $B$ is a cutoff on the $L^{2}$-norm of $u$. Hence, after normalization, one expects $u, D_{x}^{-1} n, \widetilde{V}$ to be, essentially sf.eaking, ranging in the Wiener space. This explains the choice of assumptions made in Theorem 1. One may then repeat the technique used in [Bo3] for the nonlinear Schrödinger equation $i u_{t}+u_{x x}+u|u|^{p-2}=0$ (especially when $p>4$ ) to show that $(0.1)$ is globally wellposed on a set of data $(\varphi, a, b)$ carrying the Gibbs measure which is invariant under the flow.

Theorem 3. The Zakharov system $(0.1)$ is globally wellposed on a $K_{o}$-subset $\mathcal{K}$ in $\cap_{s<\frac{1}{2}}\left(H^{s} \times H^{s-1} \times H^{s-2}\right)$ which carries the normalized Gibbs measure (0.3). Moreover $\mathcal{K}$ and the measure are invariant under the flow.

The analysis used in proving Theorem 1 is mainly an application of the contraction principle with norms e:cpressed in terms of the Fourier transform of $u$. These norms are closely related to those used in $\left[\mathrm{Bo}_{1,2}\right]$ for the NLS and KdV equations. The fixpoint problem is setup in terms of the function $u$. The first equation in (0.1) yields

$$
u(t)=S(t) \phi+i \int_{0}^{t} S(t-\tau)(n u)(\tau) d \tau \text { with } S(t)=e^{i t \partial_{x}^{2}}
$$

and $n$ may be expressed from the second equation as

$$
n=\square^{-1} \Delta\left(|u|^{2}\right)+W(t)(a, b)
$$

where $W(t)(a, b)$ is the solution of

$$
\left\{\begin{array}{l}
n_{t t}-n_{x x}=0 \\
n(x, 0)=a(x) \text { and } \quad \partial_{t} n(x, 0)=b(x)
\end{array}\right.
$$

and $\square^{-1} F$ solves

$$
\left\{\begin{array}{l}
n_{t t}-n_{x x}=F \\
n(x, 0)=0 \text { and } \partial_{t} n(x, 0)=0
\end{array}\right.
$$

Hence (0.4), (0.5) yield

$$
u(t)=S(t) \phi+i \int_{0}^{t} S(t-\tau)\left[u\left(\square^{-1} \Delta|u|^{2}\right)(\tau)+u(\tau) W(\tau)(a, b)\right] d \tau
$$

Theorem 2 for instance may then be proved by solving the fixpoint problem corresponding to (0.8). In dealing with Theorem 1 there seems to be a difficulty with this approach due to the rough conditions on the Iata $a$ and $b$. For this purpose we rely on a variant of the preceding which consists in modifying slightly the linear equation $i u_{t}+u_{x x}=0$, adding to $i u_{t}+u_{x x}$ a part of the term $u W(t)(a, b)$. (Such constructions appear in $\left[\mathrm{Bo}_{2}\right]$.) We then write

$$
i u_{t}+u_{x x}-L u=u \square^{-1}\left(|u|^{2}\right)_{x x}+[u W(t)(a, b)-L(u)]
$$

Here $L$ is a certain linear operator depending on $a, b$. The left (resp. right) member in (0.9) yield now the new linear equation (resp. perturbative term) and one applies the fixpoint method to the corresponding integral 
equation. The modification of the linear part leads only to minor technical changes while the substraction of $L(u)$ in the right member removes certain undesirable terms.

\section{Expressing in Fourier transform}

Consider the two equations

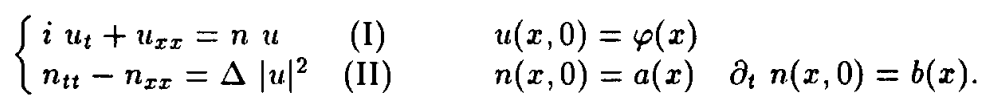

Applying Duhamel's formula to (I) yields

$$
u(t)=S(t) \varphi-i \int_{0}^{t} S(t-\tau)(n u)(\tau) d \tau
$$

and rewriting the integral term using Fourier transform gives

$$
\sum_{k} e^{i k x} \int d \lambda\left\{\widehat{n u}(k, \lambda) \frac{e^{-i k^{2} t}-e^{i \lambda t}}{\lambda+k^{2}}\right\}
$$

where

$$
\widehat{n u}(k, \lambda)=\sum_{k^{1}+k_{3}=k} \int_{\lambda^{1}+\lambda_{3}=\lambda} \widehat{n}\left(k^{1}, \lambda^{1}\right) \hat{u}\left(k_{3}, \lambda_{3}\right) .
$$

The time $t$ is restricted here to an interval $[0, T]$.

Solving (II) in $n$

$$
\left\{\begin{array}{l}
n_{t t}-n_{x x}=0 \\
n(x, 0)=a, \partial_{t} n(x, 0)=b
\end{array}\right.
$$

yields

$$
n=\sum_{k} c_{k} e^{i k(x+t)}+\sum_{k} d_{k} e^{i k(x-t)} \equiv W(t)(a, b)
$$

where

$$
\left\{\begin{array} { l } 
{ c _ { k } + d _ { k } = \widehat { a } ( k ) } \\
{ c _ { k } - d _ { k } = \frac { \widehat { b } ( k ) } { i k } }
\end{array} \Rightarrow \left\{\begin{array}{l}
c_{k}=\frac{1}{2} \widehat{a}(k)+\frac{1}{2 i k} \widehat{b}(k) \\
d_{k}=\frac{1}{2} \widehat{a}(k)-\frac{1}{2 i k} \widehat{b}(k)
\end{array}\right.\right.
$$

(assume $\widehat{b}(0)=0$ )

$$
\left\{\begin{array}{l}
n_{t t}-n_{x x}=F \\
n(x, 0)=\partial_{t} n(x, 0)=0
\end{array}\right.
$$

yields

$$
n=\sum_{k} \int d \lambda e^{i(k x+\lambda t)} \frac{\widehat{F}(k, \lambda)}{\lambda^{2}-k^{2}}-W(t)\left(h_{0}, h_{1}\right)
$$

where

$$
\left\{\begin{array}{l}
h_{0}=\sum_{k} \int d \lambda e^{i k x} \frac{\widehat{F}(k, \lambda)}{\lambda^{2}-k^{2}} \\
h_{1}=i \sum_{k} \int d \lambda e^{i k x} \frac{\lambda \cdot \widehat{F}(k, \lambda)}{\lambda^{2}-k^{2}}
\end{array}\right.
$$


a:ssurne $\hat{F}(0, \cdot)=0)$.

Hence one gets for the solution to (1.4)

$$
n \equiv \square^{-1} F=\sum_{k} e^{i k x} \int d \lambda\left\{e^{i \lambda t}-\frac{1}{2}\left(1+\frac{\lambda}{k}\right) e^{i k t}-\frac{1}{2}\left(1-\frac{\lambda}{k}\right) e^{-i k t}\right\} \frac{\widehat{F}(k, \lambda)}{\lambda^{2}-k^{2}} .
$$

For $\lambda \approx k$ write for the integrand

$$
\begin{aligned}
& {\left[e^{i \lambda t}-e^{i k t}+\frac{1}{2}\left(1-\frac{\lambda}{k}\right)\left(e^{i k t}-e^{-i k t}\right)\right] \frac{\widehat{F}(k, \lambda)}{\lambda^{2}-k^{2}} } \\
= & {\left[\frac{i e^{i k t} 0(t)}{\lambda+k}+0\left(\frac{1}{k|\lambda+k|}\right)\left(e^{i k t}-e^{-i k t}\right)\right] \hat{F}(k, \lambda) . }
\end{aligned}
$$

The same observation holds for $\lambda \approx-k$.

Fecall that $t$ is bounded by $T$. From the preceding, one may restrict the range of $\lambda$ to $\|\lambda|-| k\|>1$ (cf. $\left.\left.\mid 3 o_{1}\right]\right)$. Otherwise the interpretation of the denominator $\lambda^{2}-k^{2}$ is $k$ or $-k$ and the estimates for $\|\lambda|-| k\|>1$ capture in particular terms of the form

$$
\sum_{k} e^{i k(x+t)} \frac{1}{k} \int_{\||\lambda|-|k| \mid \leq 1} \hat{F}(k, \lambda)
$$

The Solution to (II) leads thus to following contributions for $n$

$$
\begin{gathered}
\sum k^{2} e^{i k x} \int_{\|\lambda|-| k\| \geq 1} d \lambda e^{i \lambda t} \frac{\widehat{|u|^{2}}(k, \lambda)}{\lambda^{2}-k^{2}} \\
\sum k e^{i k(x \pm t)} \int_{\| \lambda|-| k \mid \geq 1} d \lambda \frac{\widehat{|u|^{2}}(k, \lambda)}{\lambda \mp k} \\
\sum\left(\hat{a}(k) \pm \frac{1}{i k} \widehat{b}(k)\right) e^{i k(x \pm t)} \text { hence } \sum c_{k}^{ \pm} e^{i k(x \pm t)}
\end{gathered}
$$

where

$$
\sup _{k}|k|^{-o} c_{k}^{ \pm}<\infty
$$

Sulstitution in (I) yields thus following contributions to the right member of (0.8)

$$
\begin{gathered}
S(t) \varphi=\sum \hat{\varphi}(k) e^{i\left(k x+k^{2} t\right)} \\
\sum_{k} e^{i\left(k x-k^{2} t\right)}\left\{\sum_{k_{1}+k_{2}+k_{3}=k} \int d \lambda_{1} d \lambda_{2} d \lambda_{3}\right. \\
\left.\frac{\left(k_{1}+k_{2}\right)^{2}}{\left[\left(\lambda_{1}+\lambda_{2}\right)^{2}-\left(k_{1}+k_{2}\right)^{2}\right]\left(\lambda_{1}+\lambda_{2}+\lambda_{3}+k^{2}\right)} \widehat{u}\left(k_{1}, \lambda_{1}\right) \overline{\widehat{u}\left(-k_{2},-\lambda_{2}\right)} \widehat{u}\left(k_{3}, \lambda_{3}\right)\right\}
\end{gathered}
$$




$$
\begin{aligned}
& \sum_{k} \int d \lambda e^{i(k x+\lambda t)} \sum_{k_{1}+k_{2}+k_{3}=k} \int_{\lambda_{1}+\lambda_{2}+\lambda_{3}=\lambda} \\
& \frac{\left(k_{1}+k_{2}\right)^{2}}{\left[\left(\lambda_{1}+\lambda_{2}\right)^{2}-\left(k_{1}+k_{2}\right)^{2}\right]} \frac{1}{\left(\lambda+k^{2}\right)} \widehat{u}\left(k_{1}, \lambda_{1}\right) \overline{\widehat{u}\left(-k_{2},-\lambda_{2}\right)} \widehat{u}\left(k_{3}, \lambda_{3}\right) \\
& \sum_{k} e^{i\left(k x+k^{2} t\right)} \sum_{k_{1}+k_{2}+k_{3}=k} \int d \lambda_{1} d \lambda_{2} d \lambda \\
& \frac{\left(k_{1}+k_{2}\right)}{\left[\left(\lambda_{1}+\lambda_{2}\right) \pm\left(k_{1}+k_{2}\right)\right]} \frac{1}{\left(\lambda+k^{2}\right)} \widehat{u}\left(k_{1}, \lambda_{1}\right) \mid \overline{\widehat{u}\left(-k_{2},-\lambda_{2}\right)} \widehat{u}\left(k_{3}, \lambda-k_{1}-k_{2}\right) \\
& \sum_{k} \int d \lambda e^{i(k x+\lambda t)} \sum_{k_{1}+k_{2}+k_{3}=k} \int d \lambda_{1} d \lambda_{2} \\
& \frac{\left(k_{1}+k_{2}\right)}{\left[\left(\lambda_{1}+\lambda_{2}\right) \pm\left(k_{1}+k_{2}\right)\right]} \frac{1}{\left(\lambda+k^{2}\right)} \widehat{u}\left(k_{1}, \lambda_{1}\right) \overline{\widehat{u}\left(-k_{2},-\lambda_{2}\right)} \widehat{u}\left(k_{3}, \lambda-k_{1}-k_{2}\right) \\
& \sum_{k} e^{i\left(k x-k^{2} t\right)}\left[\sum_{k^{1}+k_{3}=k} c_{k^{1}}^{\mp}\left(\int \frac{\widehat{u}\left(k_{3}, \lambda \pm k^{1}\right)}{\left[\lambda+k^{2}\right]} d \lambda\right)\right] \\
& \sum_{k} \int d \lambda e^{i(k x+\lambda t)}\left[\sum_{k^{1}+k_{3}=k} c_{k^{1}}^{\mp} \widehat{u}\left(k_{3}, \lambda \pm k^{1}\right) \frac{1}{\left[\lambda+k^{2}\right]}\right] .
\end{aligned}
$$

Here all denominator factors are kept away from zero and $\left(\lambda_{1}+\lambda_{2}\right)^{2}-\left(k_{1}+k_{2}\right)^{2}$ is considered as the product $\left[\left(\lambda_{1}+\lambda_{2}\right)+\left(k_{1}+k_{2}\right)\right]\left[\left(\lambda_{1}+\lambda_{2}\right)-\left(k_{1}+k_{2}\right)\right]$.

For a fixed time interval $[0, T]$, the norm of $u$ is defined as

$$
\|u\|=\left[\sum_{k}|k|^{2 s} \int d \lambda\left(\left|\lambda+k^{2}\right|+1\right)|\widehat{u}(k, \lambda)|^{2}\right]^{1 / 2}+\sup _{k}|k|^{s_{1}}\left(\int d \lambda\left(1+\left|\lambda+k^{2}\right|\right)|\widehat{u}(k, \lambda)|^{2}\right)^{1 / 2}
$$

(where $s, s_{1}$ are the exponents from Theorem 1). In fact ( $\mathrm{cf}$. $\left[\mathrm{Bo}_{1}\right]$ ), we consider (1.17) as a restriction norm, taking the infimum of (1.17) over all representations $u=\sum_{k} \int d \lambda \widehat{u}(k, \lambda) e^{i(k x+\lambda t)}$ valid on $\mathbf{T} \times[0, T]$.

The contributions of (1.11)-(1.16) to (1.17) are estimated by

$$
\begin{gathered}
\sum_{k=k_{1}+k_{2}+k_{3}} \int d \lambda_{1} d \lambda_{2} d \lambda_{3} \\
\frac{|k|^{s}\left|k_{1}+k_{2}\right|^{2}}{\left|\left(\lambda_{1}+\lambda_{2}\right)^{2}-\left(k_{1}+k_{2}\right)^{2}\right|\left|\lambda_{1}+\lambda_{2}+\lambda_{3}+k^{2}\right|}\left|\widehat{u}\left(k_{1}, \lambda_{1}\right)\right|\left|\widehat{u}\left(-k_{2},-\lambda_{2}\right)\right|\left|\widehat{u}\left(k_{3}, \lambda_{3}\right)\right| d(k)
\end{gathered}
$$




$$
\sum_{k=k_{1}+k_{2}+k_{3}} \int_{\lambda=\lambda_{1}+\lambda_{2}+\lambda_{3}}
$$

$$
\frac{|k|^{s}\left|k_{1}+k_{2}\right|^{2}}{\left|\left(\lambda_{1}+\lambda_{2}\right)^{2}-\left(k_{1}+k_{2}\right)^{2}\right|\left|\lambda+k^{2}\right|^{1 / 2}}\left|\widehat{u}\left(k_{1}, \lambda_{1}\right)\right|\left|\widehat{u}\left(-k_{2},-\lambda_{2}\right)\right|\left|\widehat{u}\left(k_{3}, \lambda_{3}\right)\right| d(k, \lambda)
$$

$$
\sum_{k=k_{1}+k_{2}+k_{3}} \int d \lambda_{1} d \lambda_{2} d \lambda
$$

$$
\frac{|k|^{s}\left|k_{1}+k_{2}\right|}{\left|\left(\lambda_{1}+\lambda_{2}\right) \pm\left(k_{1}+k_{2}\right)\right|\left|\lambda+k^{2}\right|}\left|\widehat{u}\left(k_{1}, \lambda_{1}\right)\right|\left|\widehat{u}\left(-k_{2},-\lambda_{2}\right)\right|\left|\widehat{u}\left(k_{3}, \lambda-k_{1}-k_{2}\right)\right| d(k)
$$

$$
\sum_{k=k_{1}+k_{2}+k_{3}} \int d \lambda_{1} d \lambda_{2} d \lambda
$$

$$
\frac{|k|^{s}\left|k_{1}+k_{2}\right|}{\left|\left(\lambda_{1}+\lambda_{2}\right) \pm\left(k_{1}+k_{2}\right)\right|\left|\lambda+k^{2}\right|^{1 / 2}}\left|\widehat{u}\left(k_{1}, \lambda_{1}\right)\right|\left|\widehat{u}\left(-k_{2},-\lambda_{2}\right)\right|\left|\widehat{u}\left(k_{3}, \lambda-k_{1}-k_{2}\right)\right| d(k, \lambda)
$$

$$
\begin{gathered}
\sum_{k=k^{1}+k_{3}} \int d \lambda \frac{|k|^{s}}{\left|\lambda+k^{2}\right|}\left|c_{k^{1}}^{\mp}\right|\left|\widehat{u}\left(k_{3}, \lambda \pm k^{1}\right)\right| d(k) \\
\sum_{k=k^{1}+k_{3}} \int d \lambda \frac{|k|^{s}}{\left|\lambda+k^{2}\right|^{1 / 2}}\left|c_{k^{1}}^{\mp}\right|\left|\widehat{u}\left(k_{3}, \lambda \pm k^{1}\right)\right| d(k, \lambda)
\end{gathered}
$$

and the corresponding expressions (1.18)'-(1.23)' where the sum over $k$ is replaced by sup and $s$ by $s_{1}$. In (1.18)-(1.23), $\{d(k)\},\{d(k, \lambda)\}$ are positive satisfying

$$
\sum d(k)^{2} \leq 1 \text { and } \sum_{k} \int d \lambda d(k, \lambda)^{2} \leq 1
$$

The $\left\{c_{k^{1}}\right\}$ satisfy sup $\left|k^{1}\right|^{-\sigma}\left|c_{k^{1}}\right|<\infty$.

Before starting the estimates, we specify the operator $L u$ in $(0.9)$ and the formulas corresponding to the right member of $(0.9)$.

Write

$$
W(t)(a, b)=\sum_{k} c_{k}^{+} e^{i k(x+t)}+\sum c_{k}^{-} e^{i k(x-t)}
$$

and define $L u=L_{+} u+L_{-} u$ with

$$
\begin{aligned}
& L_{+} u=\sum_{k} c_{-2 k-1}^{+} e^{i(-k-1) x} e^{i(-2 k-1) t} \widehat{u}(k)(t)+c_{0}^{+} \widehat{u}(k)(t) \\
& L_{-} u=\sum_{k} c_{-2 k+1}^{-} e^{i(-k+1) x} e^{i(2 k-1) t} \widehat{u}(k)(t)+c_{0}^{-} \widehat{u}(k)(t)
\end{aligned}
$$


where $\widehat{u}(k)$ denote the $k$-th Fourier coefficient of $u$ with respect to the $x$-variable.

The effect of substracting $L u$ in the right member of $(0.9)$ is to eliminate the $k^{1}=-2 k_{3} \pm 1$ term and the $k^{1}=0$ term in the sums (1.22), (1.23).

Write the right member of $(0.9)$ as

$$
\sum_{k} \cdot \omega_{k}(t) e^{i\left(k x-k^{2} t\right)}
$$

If $u$ in the left member of $(0.9)$ is written as

$$
u(x, t)=\sum_{k} \Omega_{k}(t) e^{i\left(k x-k^{2} t\right)}
$$

one determines the $\Omega_{k}$ from the equations

$$
\begin{gathered}
i \Omega_{k}^{\prime} e^{-i k^{2} t}+\left(c_{0}^{+}+c_{0}^{-}\right) \Omega_{k} e^{-i k^{2} t}+c_{2 k+1}^{+} e^{i(2 k+1) t} e^{-i(k+1)^{2} t} \Omega_{-k-1}+ \\
c_{2 k-1}^{-} e^{i(-2 k+1) t} e^{-i(k-1)^{2} t} \Omega_{-k+1}=e^{-i k^{2} t} \omega_{k}
\end{gathered}
$$

hence

$$
i \Omega_{k}^{\prime}+\left(c_{0}^{+}+c_{0}^{-}\right) \Omega_{k}+c_{2 k+1}^{+} \Omega_{-k-1}+c_{2 k-1}^{-} \Omega_{-k+1}=\omega_{k} .
$$

We next discuss the (infinite dimensional) system (1.30).

Consider the selfadjoint operators $\mathcal{G}_{+}, \mathcal{G}_{-}$defined by

$$
\begin{cases}\mathcal{G}_{+}^{k j}=c_{2 k+1}^{+} & \text {if } j=-k-1 \text { and } \mathcal{G}_{+}^{k j}=0 \text { otherwise } \\ \mathcal{G}_{-}^{k j}=c_{2 k-1}^{-} & \text {if } j=-k+1 \text { and } \mathcal{G}_{-}^{k j}=0 \text { otherwise. }\end{cases}
$$

Since $\left\{c_{k}^{+}\right\},\left\{c_{k}^{-}\right\}$are Fourier coefficients of a real functions, one has indeed $c_{-k}^{ \pm}=\overline{c_{k}^{ \pm}}$(cf. (1.3)). Recall also that $\left|c_{k}^{ \pm}\right|=0\left(|k|^{\sigma}\right)$ for some small $\sigma>0$.

One rewrites then $(1.30)$ as

$$
i \Omega^{\prime}+\left(c_{0}^{+}+c_{0}^{-}\right) \Omega+\left(\mathcal{G}_{+}+\mathcal{G}_{-}\right) \Omega=\omega
$$

Considering the symmetric operator $U=\left(c_{0}^{+}+c_{0}^{-}\right) I+\mathcal{G}_{+}+\mathcal{G}_{-}$, the solution of

$$
i \Omega_{0}^{\prime}+\cup \Omega_{0}=0 \quad ; \quad \Omega_{0}(0)=\Phi \quad\left(\Phi_{k}=\widehat{\varphi}(k)\right)
$$

is formally given by

$$
\Omega_{0}=e^{i t U} \Phi
$$

and for (1.32) we get from Duhamel's formula

$$
\Omega(t)=e^{i t U} \Phi-i \int_{0}^{t} e^{i(t-\tau) U} \omega(\tau) d \tau
$$

The estimates made next will justify these formulas.

Let $I$ be an interval in $\mathbf{Z}$ centered around $k_{0}$ of length $0\left(\left|k_{0}\right|\right)$ and denote $J=I \cup(-I)$. For $r$, define $J^{r}=J+[-r, r]($ an $r$-neighborhood of $J)$. For a subset $A$ of $\mathbf{Z}$, let $P_{A}: \ell^{2}(\mathbf{Z}) \rightarrow \ell^{2}(\mathbf{Z})$ stand for the corresponding coordinate restriction. 
It is clear from the definition of $U$ that $P_{J} \cup=P_{J} \cup P_{J^{1}}$ and hence

$$
\begin{gathered}
P_{J} \cup^{r}=P_{J} \cup P_{J 1} \cup P_{J^{2}} \ldots P_{J^{r-1}} \cup P_{J r}=P_{J}\left(P_{J r} \cup P_{J r}\right)^{r} \\
\left\|P_{J} \cup^{r}\right\| \leq\left\|P_{J r} \cup P_{J r}\right\|^{r} \leq\left(\left|k_{0}\right|+r\right)^{o r}
\end{gathered}
$$

From (1.35), it is clear that for $|t|<T$, there is following tail estimate in the expansion

$$
P_{J} e^{i t \cup}=\sum_{r=0}^{\infty} \frac{(i t)^{r}}{r !} P_{J} \cup^{r} \quad \text { namely } \sum_{r>r_{1}} \frac{T^{r}}{r !}\left(\left|k_{0}\right|+r\right)^{\sigma r}<\left|k_{0}\right|^{-10} \text { for } r_{1}=0\left(T\left|k_{0}\right|^{\sigma}\right)
$$

Thus for $r_{1} \sim\left|k_{0}\right|^{\sigma}$, one has denoting $J^{r_{1}}$ by $\tilde{J}$

$$
\left\|P_{J} e^{i t \cup}-P_{J} e^{i t(P \sim \widetilde{I} \cup P \sim \mathcal{J})}\right\|<\left|k_{0}\right|^{-10}
$$

Since $e^{i t\left(P_{\widetilde{J}} \cup P_{\vec{J}}\right)}$ is unitary on $\ell^{2}(\mathbf{Z}),(1.37)$ implies

$$
\left\|P_{J} e^{i t \cup} \Phi\right\| \leq\left\|P_{\widetilde{J}} \Phi\right\|+c\left|k_{0}\right|^{-10}\|\Phi\|
$$

It is now easy to deduce from (1.38) that for $s, s_{1} \geq 0$

$$
\begin{gathered}
\sup _{k}|k|^{s_{1}}\left|\left(e^{i t \cup} \Phi\right)_{k}\right| \leq \sup _{k}\left[|k|^{s_{1}+o}\left|\Phi_{k}\right|\right]+\|\phi\| \\
\left(\sum|k|^{2 s}\left|\left(e^{i t \cup} \Phi\right)_{k}\right|^{2}\right)^{1 / 2} \leq c\left(\sum|k|^{2 s}\left|\phi_{k}\right|^{2}\right)^{1 / 2} .
\end{gathered}
$$

We now analyze the second term in (1.33)

$$
P_{J}\left(\int_{0}^{t} e^{i(t-\tau) U} \omega(\tau) d \tau\right)
$$

Write with previous notations

$$
P_{J} e^{i s U}=P_{J} e^{i s\left(P_{\mathcal{J}} \cup P_{\tilde{J}}\right)}+\sum_{r>r_{1}} \frac{(i s)^{r}}{r !} A_{r}
$$

where

$$
A_{r}=P_{J}\left(P_{J} \cup P_{J}\right)^{r}-P_{J}\left(P_{\widetilde{J}} \cup P_{\widetilde{J}}\right)^{r}
$$

hence

$$
\left\|A_{r}\right\|<\left(\left|k_{0}\right|+r\right)^{o r}
$$

let $\left\{\lambda_{\alpha}\right\}$ be the eigenvalues of $P_{\widetilde{J}} \cup P_{\widetilde{J}}$ considered as operator on $\ell_{\widetilde{J}}^{2}$ and $\left\{\xi_{\alpha}\right\}$ the corresponding normalized eigenvectors. Let $\lambda_{*}=\max \left|\lambda_{\alpha}\right|$. Hence

$$
\lambda_{*}=\left\|P_{\widetilde{J}} \cup P_{\widehat{J}}\right\| \leq\left|k_{0}\right|^{o}
$$


Write $P_{\widetilde{J}} \omega(t)=\sum_{\alpha} \nu_{\alpha}(t) \xi_{\alpha}$. Thus $\left\{\nu_{\alpha}(t)\right\}$ (resp. $\left.\left\{\widehat{\nu}_{\alpha}(\lambda)\right\}\right)$ is obtained from $\left\{\omega_{k}(t) \mid k \in \widetilde{J}\right\}$ (resp. $\left.\left\{\widehat{\omega}_{k}(\lambda)\right\}\right)$ by applying an orthogonal transformation on $\ell_{\widetilde{J}}^{2}$.

From (1.42)

$$
(1.41)=P_{J}\left(\int_{0}^{t} e^{i(t-\tau)\left(P_{\widetilde{J}} \cup P_{\widetilde{J}}\right)} \omega(\tau) d \tau\right)+\sum_{r>r_{1}} \frac{i^{r}}{r !} A_{r}\left(\int_{0}^{t}(t-\tau)^{r} \omega(\tau) d \tau\right)=(1.46)+(1.47)
$$

From the preceding, we may write for the first term

$$
(1.46)=\sum_{\alpha} P_{J} \xi_{\alpha} \cdot \int_{0}^{t} e^{i(t-\tau) \lambda_{\alpha}} \nu_{\alpha}(\tau) d \tau=\sum_{\alpha}\left(P_{J} \xi_{\alpha}\right) \cdot \int \widehat{\nu}_{\alpha}(\lambda) \frac{e^{i \lambda t}-e^{i \lambda_{\alpha} t}}{\lambda-\lambda_{\alpha}} d \lambda .
$$

Splitting the integral

$$
\int \widehat{\nu}_{\alpha}(\lambda) \frac{e^{i \lambda t}-e^{i \lambda_{\alpha} t}}{\lambda-\lambda_{\alpha}} d \lambda=\int_{|\lambda|<2 \lambda .}+\int_{|\lambda| \geq 2 \lambda}
$$

one gets $(1.46)=(1.50)+(1.51)$.

By (1.45) and the preceding

$$
\begin{aligned}
\left\|D_{t}^{1 / 2}(1.50)\right\|_{L_{i}^{2} \ell_{\mathbf{2}}^{2}} & \leq c\left|k_{0}\right|^{\sigma}\left(\sum_{\alpha} \int_{|\lambda|<2 \lambda_{\bullet}}\left|\widehat{\nu}_{\alpha}(\lambda)\right|^{2}\right)^{1 / 2}=c\left|k_{0}\right|^{\sigma}\left(\int_{|\lambda|<2 \lambda_{*}} \sum_{k \in \widetilde{J}}\left|\widehat{\omega}_{k}(\lambda)\right|^{2}\right)^{1 / 2} \\
& \leq c\left|k_{0}\right|^{\frac{3}{2} \sigma}\left(\sum_{k \in \widetilde{J}} \int d \lambda \frac{\left|\widehat{\omega}_{k}(\lambda)\right|^{2}}{1+|\lambda|}\right)^{1 / 2} .
\end{aligned}
$$

For (1.51), we have

$$
\begin{aligned}
D^{1 / 2}(1.51) & =\sum_{\alpha}\left(P_{J} \xi_{\alpha}\right) \cdot \int_{|\lambda|>2 \lambda_{\bullet}} d \lambda\left[\widehat{\nu}_{\alpha}(\lambda) \frac{|\lambda|^{1 / 2}}{\lambda-\lambda_{\alpha}} e^{i \lambda t}\right] \\
& -\sum_{\alpha}\left(P_{J} \xi_{\alpha}\right)\left[\int_{|\lambda|>2 \lambda_{\bullet}} \frac{\hat{\nu}_{\alpha}(\lambda)}{\lambda-\lambda_{\alpha}} d \lambda\right]\left(1+\left|\lambda_{\alpha}\right|\right)^{1 / 2} e^{i \lambda_{\alpha} t} \\
& =(1.53)+(1.54) .
\end{aligned}
$$

(One multiplies (1.54) with a bump function localizing in time.)

Thus clearly

$$
\|(1.53)\|_{L_{i}^{2} \ell_{\underline{z}}^{2}} \leq c\left(\sum_{\alpha} \int d \lambda \frac{\left|\widehat{\nu}_{\alpha}(\lambda)\right|^{2}}{1+|\lambda|}\right)^{1 / 2}=c\left(\int \frac{d \lambda}{1+|\lambda|} \sum_{k \in \widehat{J}}\left|\widehat{\omega}_{k}(\lambda)\right|^{2}\right)^{1 / 2}
$$

and 


$$
\begin{aligned}
\|(1.54)\|_{L_{i}^{2} \ell_{\mathbf{z}}^{2}} & \leq \lambda_{*}^{1 / 2}\left[\sum_{\alpha}\left|\int_{|\lambda|>2 \lambda_{*}} d \lambda \hat{\nu}_{\alpha}(\lambda) \frac{1}{\lambda}\left(1+\frac{\lambda_{\alpha}}{\lambda}+\left(\frac{\lambda_{\alpha}}{\lambda}\right)^{2}+\cdots\right)\right|^{2}\right]^{1 / 2} \\
& \leq c\left|k_{0}\right|^{\sigma / 2} \sum_{s \geq 0} \lambda_{*}^{s}\left[\sum_{\alpha}\left|\int_{|\lambda|>2 \lambda_{*}} \widehat{\nu}_{\alpha}(\lambda) \frac{d \lambda}{\lambda^{s+1}}\right|^{2}\right]^{1 / 2} \\
& =c\left|k_{0}\right|^{\sigma / 2} \sum_{s \geq 0} \lambda_{*}^{s}\left[\sum_{k \in \widetilde{J}}\left|\int_{|\lambda|>2 \lambda_{*}} \widehat{\omega}_{k}(\lambda) \frac{d \lambda}{\lambda^{s+1}}\right|^{2}\right]^{1 / 2} \\
& \leq c\left|k_{0}\right|^{\sigma / 2} \sum_{s \geq 0} 2^{-s}\left[\left.\sum_{k \in \widetilde{J}}\left|\int_{|\lambda|>2 \lambda_{*}}\right| \widehat{\omega}_{k}(\lambda)\left|\frac{d \lambda}{|\lambda|}\right|^{2}\right|^{1 / 2}\right. \\
& =c\left|k_{0}\right|^{\sigma / 2}\left[\sum_{k \in \widetilde{J}}\left(\int \frac{\left|\hat{\omega}_{k}(\lambda)\right|}{1+|\lambda|} d \lambda\right)^{2}\right]^{1 / 2} .
\end{aligned}
$$

Collecting (1.52), (1.55), (1.56)

$$
\left\|D^{1 / 2}(1.46)\right\|_{L_{i}^{2} \ell_{\mathbf{Z}}^{2}} \leq c\left|k_{0}\right|^{\frac{3}{2} \sigma}\left(\sum_{k \in \widetilde{J}} \int d \lambda \frac{\left|\widehat{\omega}_{k}(\lambda)\right|^{2}}{1+|\lambda|}\right)^{1 / 2}+c\left|k_{0}\right|^{\sigma / 2}\left[\sum_{k \in \widetilde{J}}\left[\int \frac{\left|\widehat{\omega}_{k}(\lambda)\right|}{1+|\lambda|} d \lambda\right]^{2}\right]^{1 / 2} .
$$

For the second term (1.47), one gets by partial integration

$$
\sum_{r>r_{1}} \frac{i^{r}}{r !} A_{r}\left[-t^{r} D^{-1} \omega(0)-r \int_{0}^{t} D^{-1} \omega(\tau)(t-\tau)^{r-1} d \tau\right]
$$

from where clearly (after again localizing in time)

$$
\|(1.47)\|_{L_{t}^{2} \ell_{\mathbf{Z}}^{2}} \leq c\left(\sum_{r>r_{1}} \frac{\left\|A_{r}\right\|}{r !}\right)\left\|D^{-1} \omega\right\|_{L_{\ell}^{\infty} \ell_{\mathbf{Z}}^{2}} \leq\left|k_{0}\right|^{-10}\left(\sum_{k}\left[\int \frac{\left|\widehat{\omega}_{k}(\lambda)\right|}{1+|\lambda|}\right]^{2}\right)^{1 / 2}
$$

by (1.44) and the choice of $r_{1}$. The same estimate (1.59) holds for $\left\|D^{1 / 2}(1.47)\right\|_{L_{1}^{2} \ell_{2}^{2}}$. Consequently, by (1.57), (1.59)

$$
\begin{aligned}
\left\|D^{1 / 2}(1.41)\right\|_{L_{i}^{2} \ell_{\mathbf{z}}^{2}} \leq & c\left|k_{0}\right|^{\frac{3}{2} \sigma}\left[\sum_{k \in \widetilde{J}}\left(\int d \lambda \frac{\left|\widehat{\omega}_{k}(\lambda)\right|^{2}}{1+|\lambda|}\right)+\left(\int \frac{\left|\widehat{\omega}_{k}(\lambda)\right|}{1+|\lambda|}\right)^{2}\right]^{1 / 2} \\
& +\left|k_{0}\right|^{-10}\left[\sum_{k}\left(\int \frac{\left|\widehat{\omega}_{k}(\lambda)\right|}{1+|\lambda|}\right)^{2}\right]^{1 / 2}
\end{aligned}
$$

Similarly to (1.39), (1.40) one deduces from (1.60) that for $s, s_{1} \geq 0$ 


$$
\begin{gathered}
\sup _{k}|k|^{s_{1}}\left\|D_{t}^{1 / 2}\left[\int_{0}^{t} e^{i(t-\tau) U} \omega(\tau) d \tau\right]_{k}\right\|_{L_{t}^{2}} \leq \\
c \sup _{k}|k|^{2 \sigma+s_{1}}\left[\left(\int \frac{\left|\widehat{\omega}_{k}(\lambda)\right|^{2}}{1+|\lambda|}\right)^{1 / 2}+\int \frac{\left|\widehat{\omega}_{k}(\lambda)\right|}{1+|\lambda|}\right]+\left[\sum_{k}\left(\int \frac{\left|\widehat{\omega}_{k}(\lambda)\right|}{1+|\lambda|}\right)^{2}\right]^{1 / 2}
\end{gathered}
$$

and

$$
\begin{aligned}
& \left(\sum_{k}|k|^{2 s}\left\|D_{t}^{1 / 2}\left[\int_{0}^{t} e^{i(t-\tau) U} \omega(\tau) d \tau\right]_{k}\right\|_{L_{i}^{2}}^{2}\right)^{1 / 2} \leq \\
& \left(\sum_{k}|k|^{2(s+2 \sigma)}\left[\int \frac{\left|\widehat{\omega}_{k}(\lambda)\right|^{2}}{1+|\lambda|}+\left(\int \frac{\left|\widehat{\omega}_{k}(\lambda)\right|}{1+|\lambda|}\right)^{2}\right]\right)^{1 / 2} .
\end{aligned}
$$

Observe by (1.29) that $\widehat{u}(k, \lambda)=\widehat{\Omega}_{k}\left(\lambda+k^{2}\right)$ and hence the norm (1.17) may be rewritten as

$$
\left(\sum_{k}|k|^{2 s}\left\|D^{1 / 2} \Omega_{k}\right\|_{2}^{2}\right)^{1 / 2}+\sup _{k}\left(|k|^{s_{1}}\left\|D^{1 / 2} \Omega_{k}\right\|_{2}\right)
$$

which, by (1.33), is given by the sum of (1.39), (1.40), (1.61), (1.62).

Thus one needs $\varphi=u(x, 0)$ to satisfy

$$
\left(\sum|k|^{2 s}|\widehat{\varphi}(k)|^{2}\right)^{1 / 2}+\sup _{k}\left(|k|^{s_{1}+\sigma}|\widehat{\varphi}(k)|\right)<\infty .
$$

Recall that (1.28) is the right member of $(0.9)$, thus the expressions (1.11)-(1.16) with the modification in (1.15), (1.16) mentionned above. The estimate on $\widehat{\omega}_{k}(\lambda)$ (by putting absolute values of the Fourier transforms inside as to get (1.18)-(1.23)) and bounding (1.61), (1.62) by duality clearly yields again the expressions (1.18)-(1.23) with an extra factor $|k|^{2 \sigma}$ and deleting in (1.22), (1.23) the $k^{1}=0, k^{1}=-2 k_{3} \pm 1$ (with corresponding sign) terms.

As will follow below, there is a saving of an extra factor $|k|^{\gamma}$ (for some $\gamma>0$ ) in the estimates of (1.18)-(1.23), so that the additional $|k|^{2 \sigma}$-factor is harmless for $\sigma$ small enough. The value of $\sigma$ will depend on those of $s<\frac{1}{2}<s_{1}$.

Recall following two estimates related to the linear 1-dimensional periodic Schrödinger operator (cf. $\left[\mathrm{B}_{1}\right]$ )

$$
\begin{aligned}
& \left\|\sum_{k} \int d \lambda a(k, \lambda) e^{i(k x+\lambda t)}\right\|_{L_{\text {loc(t) }}^{4} L_{x}^{4}(\mathbf{T})} \leq c\left(\sum_{k} \int d \lambda\left|\lambda+k^{2}\right|^{3 / 4}|a(k, \lambda)|^{2}\right)^{1 / 2} \\
& \left\|\sum_{k \sim K} \int d \lambda a(k, \lambda) e^{i(k x+\lambda t)}\right\|_{L_{\text {loc(t) }}^{6} L_{x}^{\dagger}(\mathbf{T})} \ll K^{\varepsilon}\left(\sum_{k} \int d \lambda\left|\lambda+k^{2}\right||a(k, \lambda)|^{2}\right)^{1 / 2} .
\end{aligned}
$$




\section{Estimation of (1.19)}

Define

$$
\begin{aligned}
& c(k, \lambda)=\left|\lambda+k^{2}\right|^{1 / 2}|\widehat{u}(k, \lambda)| \\
& \bar{c}(k, \lambda)=|k|^{-3} c(k, \lambda) \\
& \overline{\bar{c}}(k, \lambda)=|k|^{-s_{1}} c(k, \lambda)
\end{aligned}
$$

hence

$$
\|u\|=\|\{\bar{c}(k, \lambda)\}\|_{\ell_{k}^{2} L_{\lambda}^{2}}+\|\{\overline{\bar{c}}(k, \lambda)\}\|_{\ell_{k}^{\infty} L_{\lambda}^{2}} .
$$

We have to estimate $\left({ }^{*}\right)$

$$
\sum_{k=k_{1}+k_{2}+k_{3}} \int_{\lambda=\lambda_{1}+\lambda_{2}+\lambda_{3}}
$$

$$
\frac{c\left(k_{1}, \lambda_{1}\right) c\left(k_{2}, \lambda_{2}\right) c\left(k_{3}, \lambda_{3}\right)\left(k_{1}+k_{2}\right)^{2}|k|^{s} d(k, \lambda)}{\left|\lambda_{1}+k_{1}^{2}\right|^{1 / 2}\left|\lambda_{2}-k_{2}^{1}\right|^{1 / 2}\left|\lambda_{3}+k_{3}^{2}\right|^{1 / 2}\left|\lambda+k^{2}\right|^{1 / 2}\left|\left(\lambda_{1}+\lambda_{2}\right)+\left(k_{1}+k_{2}\right)\right|\left|\left(\lambda_{1}+\lambda_{2}\right)-\left(k_{1}+k_{2}\right)\right|} .
$$

Observe that

$-\max \left(\left|\left(\lambda_{1}+\lambda_{2}\right)+\left(k_{1}+k_{2}\right)\right|,\left|\left(\lambda_{1}+\lambda_{2}\right)-\left(k_{1}+k_{2}\right)\right|\right) \geq\left|k_{1}+k_{2}\right|$

$-\max \left(\left|\lambda_{1}+k_{1}^{2}\right|^{1 / 2},\left|\lambda_{2}-k_{2}^{2}\right|^{1 / 2},\left|\left(\lambda_{1}+\lambda_{2}\right) \pm\left(k_{1}+k_{2}\right)\right|^{1 / 2}\right) \geq\left(\left|k_{1}+k_{2}\right|\left|k_{1}-k_{2} \pm 1\right|\right)^{1 / 2}$

- $\max \left(\left|\lambda_{3}+k_{3}^{2}\right|^{1 / 2},\left|\lambda+k^{2}\right|^{1 / 2},\left|\left(\lambda_{1}+\lambda_{2}\right) \pm\left(k_{1}+k_{2}\right)\right|^{1 / 2}\right) \geq\left(\left|k_{1}+k_{2}\right|\left|k_{1}+k_{2}+2 k_{3} \pm 1\right|\right)^{1 / 2}$.

Thus (2.2) yields one $\left|k_{1}+k_{2}\right|$-factor. Either (2.3) or (2.4) yield another $\left|k_{1}+k_{2}\right|$-factor, unless

$$
\left|k_{1}\right| \sim\left|k_{2}\right|
$$

and

$$
\left|k_{1}+k_{2}\right| \sim\left|k_{3}\right| .
$$

Assume $(2.5)+(2.6)$ does not hold. One estimates (2.1) by

$$
\begin{gathered}
\left(\left|k_{1}\right|+\left|k_{2}\right|+\left|k_{3}\right|\right)^{s} \frac{c\left(k_{1}, \lambda_{1}\right)}{\left|\lambda_{1}+k_{1}^{2}\right|^{1 / 2}} \frac{c\left(k_{2}, \lambda_{2}\right)}{\left|\lambda_{2}-k_{2}^{2}\right|^{1 / 2}} \frac{c\left(k_{3}, \lambda_{3}\right)}{\left|\lambda_{3}+k_{3}^{2}\right|^{1 / 2}} \frac{d(k, \lambda)}{\left|\lambda+k^{2}\right|^{1 / 2}} \\
\left(\left|\lambda_{1}+k_{1}^{2}\right|^{1 / 2}+\left|\lambda_{2}-k_{2}^{2}\right|^{1 / 2}+\left|\lambda_{3}+k_{3}^{2}\right|^{1 / 2}+\left|\lambda+k^{2}\right|^{1 / 2}\right)
\end{gathered}
$$

which may be further estimated by an $\left(L^{2}, L^{6}, L^{6}, L^{6}\right)$-bound using (1.66) as in the case of the 1-dim NLSE $\left(\mathrm{cf} .\left[\mathrm{Bo}_{1}\right]\right)\left({ }^{* *}\right)$.

In fact, considering instead of (2.3) (resp. (2.4)) $(2.3) \cdot(2.4)^{\varepsilon}$ (resp. $\left.(2.4) \cdot(2.3)^{\varepsilon}\right)$ and the possibility of replacing the $\frac{1}{2}$-denominator pówer by $\frac{1}{2}-\varepsilon$ in the $L^{6}$-inequality

$\left.{ }^{*}\right)$ Denominator expressions $|\ldots|$ will be understood as $|\ldots|+1$.

$\left({ }^{* *}\right)$ The $\left(\left|k_{1}\right|+\left|k_{2}\right|+\left|k_{3}\right|\right)^{*}$-factor is absorbed by $c\left(k_{i_{1}}, \lambda_{i_{1}}\right)$ where $\left|k_{i_{1}}\right| \geq\left|k_{i_{2}}\right| \geq\left|k_{i_{3}}\right|$. 


$$
\left\|\sum_{|k|<K} e^{i k x} \int \frac{A(k, \lambda)}{\left|\lambda-k^{2}\right|^{1 / 2-\varepsilon}+1} e^{i \lambda t} d \lambda\right\|_{L_{\text {loc }(x, t)}^{6}} \ll K^{\varepsilon^{\prime}(\varepsilon)}\left(\sum_{k} \int d \lambda|A(k, \lambda)|^{2}\right)^{1 / 2}
$$

one may gain a power $|k|^{\varepsilon}$, unless (since either $(2.5)$ or $(2.6)$ is assumed to fail)

$$
\text { (2.6) fails and } k_{1}-k_{2}=0(1)
$$

or

$$
\text { (2.5) fails and } k_{1}+k_{2}+2 k_{3}=0(1) \text {. }
$$

In both cases (2.8), (2.9) a power $|k|^{\varepsilon}$ may be gained also, using $c\left(k_{i_{2}}, \lambda_{i_{2}}\right)$ where $\left|k_{i_{1}}\right| \geq\left|k_{i_{2}}\right| \geq\left|k_{i_{3}}\right|$. Hence if $(2.5)+(2.6)$ does not hold, there is a gain of an extra $|k|^{\varepsilon}$-factor for some constant $\varepsilon>0$.

Assume next $(2.5)+(2.6)$.

Rewrite (2.1) as

$$
\frac{\left|k_{1}+k_{2}\right|^{1-s}\left|k_{1}\right|^{-s}}{\left|\left(\lambda_{1}+\lambda_{2}\right) \pm\left(k_{1}+k_{2}\right)\right|} \frac{\bar{c}\left(k_{1}, \lambda_{1}\right)}{\left|\lambda_{1}+k_{1}^{2}\right|^{1 / 2}} \frac{\bar{c}\left(k_{2}, \lambda_{2}\right)}{\left|\lambda_{2}-k_{2}^{2}\right|^{1 / 2}} \frac{\bar{c}\left(k_{3}, \lambda_{3}\right)}{\left|\lambda_{3}+k_{3}^{2}\right|^{1 / 2}} \frac{d(k, \lambda)}{\left|\lambda+k^{2}\right|^{1 / 2}} .
$$

Assume

$$
\begin{gathered}
k_{1}-k_{2}=0(1) \\
k_{1}+k_{2}+2 k_{3}=0(1)
\end{gathered}
$$

are not both valid. Then one may exploit $(2.3)^{\varepsilon}$ or $(2.4)^{\varepsilon}$ to gain $\left|k_{1}+k_{2}\right|^{\varepsilon}$ and estimate (2.10) in $\left(L^{4}, L^{4}, L^{4}, L^{4}\right)$ using the $L^{4}$-bound $(1.65)$

$$
\left\|\sum e^{i k x} \int \frac{A(k, \lambda)}{\left|\lambda-k^{2}\right|^{3 / 8}+1} e^{i \lambda t} d \lambda\right\|_{L_{\operatorname{loc}(x, t)}^{4} \leq} \leq\left(\sum_{k} \int d \lambda|A(k, \lambda)|^{2}\right)^{1 / 2}
$$

provided $s>\frac{1-\varepsilon}{2}\left(<\frac{1}{2}\right)$. In fact, there is clearly again a gain of a $|k|^{\varepsilon} \leq\left|k_{1}\right|^{\varepsilon}$ factor.

If (2.11), (2.12) hold, one has for (2.1)

$$
\sum_{k_{1}} \int_{\lambda=\lambda_{1}+\lambda_{2}+\lambda_{3}}\left|k_{1}\right|^{1+s} \frac{c\left(k_{1}, \lambda_{1}\right)}{\left|\lambda_{1}+k_{1}^{2}\right|^{1 / 2}} \frac{c\left(k_{1}, \lambda_{2}\right)}{\left|\lambda_{2}-k_{2}^{2}\right|^{1 / 2}} \frac{c\left(-k_{1}, \lambda_{3}\right)}{\left|\lambda_{3}+k_{1}^{2}\right|^{1 / 2}} \frac{d\left(k_{1}, \lambda\right)}{\left|\lambda+k^{2}\right|^{1 / 2}}
$$

which is estimated as $\left(s_{1}>\frac{1}{2}\right)$

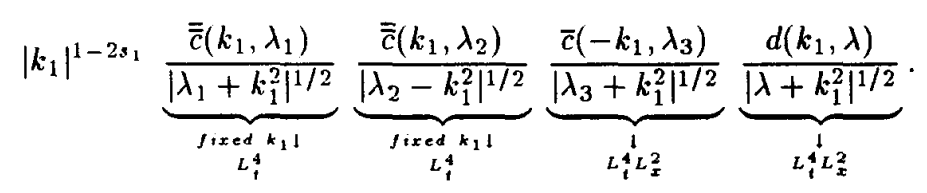

Assume $s_{1}<s+\varepsilon\left(\varepsilon>0\right.$ some number; see above). If $s_{1}>\frac{1}{2}$, the $\ell_{k}^{2} L_{\lambda}^{2}$-norm of $(2.1)$ is controlled. For the $\ell_{k}^{\infty} L_{\lambda}^{2}$-norm, one multiplies with $|k|^{s_{1}}$ instead of $|k|^{s}$. Since $s_{1}<s+\varepsilon$, previous estimates already yield the bound, except if (2.11) and (2.12) hold.

If $(2.11)+(2.12)$, one meets the condition $1+s_{1}-3 s_{1}<0$, i.e. $s_{1}>\frac{1}{2}$.

Since the estimated are not tied, one may allow an extra factor $|k|^{\sigma}, \sigma>0$, referred to at the end of previous section. 


\section{Estimation of (1.18)}

$$
\begin{gathered}
\sum_{k=k_{1}+k_{2}+k_{3}} \int_{\lambda=\lambda_{1}+\lambda_{2}+\lambda_{3}} \\
\frac{c\left(k_{1}, \lambda_{1}\right) c\left(k_{2}, \lambda_{2}\right) c\left(k_{3}, \lambda_{3}\right)\left(k_{1}+k_{2}\right)^{2}|k|^{s} d(k)}{\left|\lambda_{1}+k_{1}^{2}\right|^{1 / 2}\left|\lambda_{2}-k_{2}^{2}\right|^{1 / 2}\left|\lambda_{3}+k_{3}^{2}\right|^{1 / 2}\left|\lambda+k^{2}\right|\left|\left(\lambda_{1}+\lambda_{2}\right)+\left(k_{1}+k_{2}\right)\right|\left|\left(\lambda_{1}+\lambda_{2}\right)-\left(k_{1}+k_{2}\right)\right|}
\end{gathered}
$$

$\sum d(k)^{2}=1$.

Write, letting $\delta>0$ be arbitrarily small

$$
\frac{d(k)}{\left|\lambda+k^{2}\right|+1}=\frac{d(k)}{\left|\lambda+k^{2}\right|^{\frac{1}{2}+\delta}} \cdot \frac{1}{\left|\lambda+k^{2}\right|^{\frac{1}{2}-\delta}}
$$

and denote the first factor $d(k, \lambda)$. Thus $\sum_{k} \int d \lambda d(k, \lambda)^{2} \leq 1$.

In (2.1), the $\left|\lambda+k^{2}\right|^{1 / 2}$-factor in the denominator is then replaced by $\left|\lambda-k^{2}\right|^{\frac{1}{2}-\delta}$. This is again harmless, letting $\delta$ be sufficiently small, as appears clearly from preceding analysis.

\section{Estimation of (1.20) and (1.21)}

Consider (1.21). The modifications for (1.20) will be indicated at the end of this section.

$$
\begin{gathered}
\sum_{k=k_{1}+k_{2}+k_{3}} \int d \lambda_{1} d \lambda_{2} d \lambda \\
\frac{|k|^{s}\left|k_{1}+k_{2}\right| c\left(k_{1}, \lambda_{1}\right) c\left(k_{2}, \lambda_{2}\right) c\left(k_{3}, \lambda-k_{1}-k_{2}\right) d(k, \lambda)}{\left|\left(\lambda_{1}+\lambda_{2}\right) \pm\left(k_{1}+k_{2}\right)\right|\left|\lambda_{1}+k_{1}^{2}\right|^{1 / 2}\left|\lambda_{2}-k_{2}^{2}\right|^{1 / 2}\left|\lambda-k_{1}-k_{2}+k_{3}^{2}\right|^{1 / 2}\left|\lambda+k^{2}\right|^{1 / 2}} \\
\max \left(\left|\lambda_{1}+k_{1}^{2}\right|^{1 / 2},\left|\lambda_{2}-k_{2}^{2}\right|^{1 / 2},\left|\left(\lambda_{1}+\lambda_{2}\right) \pm\left(k_{1}+k_{2}\right)\right|^{1 / 2}\right) \geq\left(\left|k_{1}+k_{2}\right|\left|k_{1}-k_{2} \pm 1\right|\right)^{1 / 2} \\
\max \left(\left|\lambda-k_{1}-k_{2}+k_{3}^{2}\right|^{1 / 2},\left|\lambda+k^{2}\right|^{1 / 2}\right) \geq\left(\left|k_{1}+k_{2}\right|\left|k_{1}+k_{2}+2 k_{3}+1\right|\right)^{1 / 2} .
\end{gathered}
$$

Assume $\left|k_{1}\right| \geq\left|k_{2}\right|$.

(1) $\left|k_{3}\right| \not\left|k_{1}+k_{2}\right|$

Then (4.3) is a least $c\left|k_{1}+k_{2}\right|$.

(1.1) $\left|\lambda-k_{1}-k_{2}+k_{3}^{2}\right|^{1 / 2} \geq\left|k_{1}+k_{2}\right|$

Define

$$
F(x, t)=\sum_{k_{1}, k_{2}} e^{i\left(k_{1}+k_{2}\right)(x+t)}\left\{\int d \lambda_{1} d \lambda_{2} \frac{\bar{c}\left(k_{1}, \lambda_{1}\right) \bar{c}\left(k_{2}, \lambda_{2}\right)}{\left|\lambda_{1}+k_{1}^{2}\right|^{1 / 2}\left|\lambda_{2}-k_{2}^{2}\right|^{1 / 2}\left|\left(\lambda_{1}+\lambda_{2}\right) \pm\left(k_{1}+k_{2}\right)\right|}\right\}
$$




$$
\begin{aligned}
& G(x, t)=\sum_{k_{3}} \int d \lambda_{3} \bar{c}\left(k_{3}, \lambda_{3}\right) e^{i\left(k_{3} x+\lambda_{3} t\right)} \\
& H(x, t)=\sum_{k} \int d \lambda \frac{d(k, \lambda)}{\left|\lambda+k^{2}\right|^{1 / 2}} e^{i(k x+\lambda t)} .
\end{aligned}
$$

Reexpressing in terms of functions, estimate (4.1) by $\int_{\mathbf{T} \times[0,1]} F G H d x d t \leq\|F\|_{4} \cdot\|G\|_{2}\|H\|_{4}$. In fact, $\|F\|_{q}<\infty$ for $q<\infty$.

Estimate $\|F\|_{q}$ by Hausdorff-Young. Take weights $\omega(\ell) \geq 0$ s.t. $\sum \omega(\ell)^{q}<\infty$ and consider

$$
\sum_{k_{1}, k_{2}} \int d \lambda_{1} d \lambda_{2} \frac{\omega\left(k_{1}+k_{2}\right)}{\left|\lambda_{1}+k_{1}^{2}\right|^{1 / 2}\left|\lambda_{2}-k_{2}^{2}\right|^{1 / 2}\left|\left(\lambda_{1}+\lambda_{2}\right) \pm\left(k_{1}+k_{2}\right)\right|} \bar{c}\left(k_{1}, \lambda_{1}\right) \bar{c}\left(k_{2}, \lambda_{2}\right) .
$$

From Shur's lemma, it suffices to bound uniformly for fixed $\left(k_{2}, \lambda_{2}\right)$ (and vice versa)

$$
\sum_{k_{1}} \int d \lambda_{1} \frac{\omega\left(k_{1}+k_{2}\right)}{\left(\left|\lambda_{1}+k_{1}^{2}\right|^{1 / 2}+1\right)\left(\left|\lambda_{2}-k_{2}^{2}\right|^{1 / 2}+1\right)\left(\left|\left(\lambda_{1}+\lambda_{2}\right) \pm\left(k_{1}+k_{2}\right)\right|+1\right)} .
$$

Use the fact that for $0 \leq \alpha, \beta<1, \alpha+\beta>1$

$$
\int \frac{d \lambda}{\left(|\lambda-a|^{\alpha}+1\right)\left(|\lambda-b|^{\beta}+1\right)} \leq \frac{1}{(1+|a-b|)^{\alpha+\beta-1}}
$$

Hence, integrating (4.8) in $\lambda_{1}$ yields bound

$$
\sum_{k_{1}} \frac{\omega\left(k_{1}+k_{2}\right)}{\left[1+\left|\lambda_{2}-k_{1}^{2} \pm\left(k_{1}+k_{2}\right)\right|\right]^{\frac{1}{2}-}} \leq\left[\sum_{k_{1}} \frac{1}{\left[1+\left|\lambda_{2}-k_{1}^{2} \pm\left(k_{1}+k_{2}\right)\right|\right]^{\frac{q^{\prime}}{2}}-}\right]^{1 / q^{\prime}}
$$

from assumption on $\{\omega(\ell)\}$. Since for all $a \in \mathbf{R}, \alpha>\frac{1}{2}$,

$$
\sum_{k} \frac{1}{1+\left|k^{2}+a\right|^{\alpha}}<c
$$

this gives the estimate.

Remark. Considering $(4.2)^{\varepsilon}$, for some $\varepsilon>0$, and the fact that in previous estimate, the exponents of $\left|\lambda_{1}+k_{1}^{2}\right|^{1 / 2},\left|\lambda_{2}-k_{2}^{2}\right|^{1 / 2}$ or $\left|\left(\lambda_{1}+\lambda_{2}\right) \pm\left(k_{1}+k_{2}\right)\right|$ may be lowered, a factor $|k|^{\varepsilon}, \varepsilon>0$ may be saved, unless $k_{1}-k_{2}=0(1)$ in which case we use the $\left|k_{2}\right|^{3}$-factor, $\left|k_{2}\right| \sim\left|k_{1}+k_{2}\right|$, coming from $c\left(k_{2}, \lambda_{2}\right)$, for this purpose.

(1.2) $\left|\lambda+k^{2}\right|^{1 / 2} \geq\left|k_{1}+k_{2}\right|$

Redefine

$$
\begin{gathered}
G(x, t)=\sum_{k_{2}} \int d \lambda_{3} \frac{\bar{c}\left(k_{3}, \lambda_{3}\right)}{\left|\lambda_{3}+k_{3}^{2}\right|^{1 / 2}} e^{i\left(k_{3} x+\lambda_{3} t\right)} \\
H(x, t)=\sum_{k} \int d \lambda d(k, \lambda) e^{i(k x+\lambda t)}
\end{gathered}
$$

and estimate (4.1) by $\int F G H \leq\|F\|_{4}\|G\|_{4}\|H\|_{2} \leq\|u\|^{3}$ as in (1.1). 
(2) $\left|k_{3}\right| \sim\left|k_{1}+k_{2}\right|, k_{1}+k_{2}+2 k_{3} \neq 0(1)$

Then (4.3) is at least $\left|k_{1}+k_{2}\right|^{1 / 2}$ and the $\left|k_{3}\right|^{s}$-factor from $c\left(k_{3}, \lambda_{3}\right)$ gives another $\left|k_{1}+k_{2}\right|^{3}$. Hence it remains to gain $\left|k_{1}+k_{2}\right|^{\frac{1}{2}-3}\left(s<\frac{1}{2}\right.$ close to $\left.\frac{1}{2}\right)$, which may be done from (4.2), unless $k_{1}-k_{2}=0(1)$, in which case we use the $\left|k_{2}\right|^{s}$-factor from $c\left(k_{2}, \lambda_{2}\right)$. Use the same estimates as in (1).

(3) $k_{1}+k_{2}+2 k_{3}=0(1)$

Rewrite (4.1)

$$
\sum_{k_{1}, k_{2}} \int d \lambda_{1} d \lambda_{2} d \lambda
$$

$$
\frac{\left|k_{1}+k_{2}\right|^{s+1} c\left(k_{1}, \lambda_{1}\right) c\left(k_{2}, \lambda_{2}\right) c\left(-\frac{k_{1}+k_{2}}{2}, \lambda-k_{1}-k_{2}\right) d\left(\frac{k_{1}+k_{2}}{2}, \lambda\right)}{\left|\left(\lambda_{1}+\lambda_{2}\right) \pm\left(k_{1}+k_{2}\right)\right|\left|\lambda_{1}+k_{1}^{2}\right|^{1 / 2}\left|\lambda_{2}-k_{2}^{2}\right|^{1 / 2}\left|\lambda-k_{1}-k_{2}+\left(\frac{k_{1}+k_{2}}{2}\right)^{2}\right|^{1 / 2}\left|\lambda+\left(\frac{k_{1}+k_{2}}{2}\right)^{2}\right|^{1 / 2}}
$$

$\left(k_{3}=-\frac{k_{1}+k_{2}}{2}, k=\frac{k_{1}+k_{2}}{2}\right)$.

Hence

$$
\begin{gathered}
\sum_{k_{1}, k_{2}} \int d \lambda_{1} d \lambda_{2} d \lambda \\
\frac{\left|k_{1}+k_{2}\right|^{1-s_{1}} \bar{c}\left(k_{1}, \lambda_{1}\right) c\left(k_{2}, \lambda_{2}\right) \overline{\bar{c}}\left(-\frac{k_{1}+k_{2}}{2}, \lambda-k_{1}-k_{2}\right) d\left(\frac{k_{1}+k_{2}}{2}, \lambda\right)}{\left|\left(\lambda_{1}+\lambda_{2}\right) \pm\left(k_{1}+k_{2}\right)\right|\left|\lambda_{1}+k_{1}^{2}\right|^{1 / 2}\left|\lambda_{2}-k_{2}^{2}\right|^{1 / 2}\left|\lambda-k_{1}-k_{2}+\left(\frac{k_{1}+k_{2}}{2}\right)^{2}\right|^{1 / 2}\left|\lambda+\left(\frac{k_{1}+k_{2}}{2}\right)^{2}\right|^{1 / 2}}
\end{gathered}
$$

(3.1) $\left|k_{1}\right| \sim\left|k_{2}\right|$

If $\left|k_{1}\right| \sim\left|k_{2}\right|$, we may replace $\left|k_{1}+k_{2}\right|^{1-s_{1}} c\left(k_{2}, \lambda_{2}\right)$ by $\bar{c}\left(k_{2}, \lambda_{2}\right)$, assuming $s+s_{1}>1$, and estimate by $\int F_{1} \cdot G_{1} \leq\left\|F_{1}\right\|_{2} \cdot\left\|G_{1}\right\|_{2}$, where

$$
\begin{gathered}
F_{1}=\sum_{k_{1}, k_{2}} e^{i\left(k_{1}+k_{2}\right)\left(\frac{x}{2}+t\right)}\left\{\int d \lambda_{1} d \lambda_{2} \frac{\bar{c}\left(k_{1}, \lambda_{1}\right) \bar{c}\left(k_{2}, \lambda_{2}\right)}{\left|\lambda_{1}+k_{1}^{2}\right|^{1 / 2}\left|\lambda_{2}-k_{2}^{2}\right|^{1 / 2}\left|\lambda_{1}+\lambda_{2} \pm\left(k_{1}+k_{2}\right)\right|}\right\} \\
\left\{\int d \lambda_{3} e^{i \lambda_{3} t} \frac{\overline{\bar{c}}\left(-\frac{k_{1}+k_{2}}{2}, \lambda_{3}\right)}{\left|\lambda_{3}+\left(\frac{k_{1}+k_{2}}{2}\right)^{2}\right|^{1 / 2}}\right\} \\
G_{1}=\sum_{k} \int d \lambda \frac{d(k, \lambda)}{\left|\lambda+k^{2}\right|^{1 / 2}} e^{-i(k x+\lambda t)}
\end{gathered}
$$
To estimate $\left\|F_{1}\right\|_{2} \leq\left(\sum_{k}\left[\sum_{k_{1}+k_{2}=k}\{\}\right]^{2}\|\overline{\bar{c}}\|_{\ell_{k}^{\infty} L_{\lambda}^{2}}^{2}\right)^{1 / 2}$, consider $\{\omega(\ell)\}$ with $\sum \omega(\ell)^{2} \leq 1$ and the expres-
sion 


$$
\sum_{k_{1} k_{2}} \int d \lambda_{1} d \lambda_{2} \frac{\omega\left(k_{1}+k_{2}\right)}{\left|\lambda_{1}+k_{1}^{2}\right|^{1 / 2}\left|\lambda_{2}-k_{2}^{2}\right|^{1 / 2}\left|\lambda_{1}+\lambda_{2} \pm\left(k_{1}+k_{2}\right)\right|} \bar{c}\left(k_{1}, \lambda_{1}\right) \bar{c}\left(k_{2}, \lambda_{2}\right)
$$

which is bounded from the estimate of (4.7).

(3.2) $\left|k_{1}\right| \gg\left|k_{2}\right|$

Then (4.2) $\geq\left|k_{1}\right|$, hence we consider $(4.2)^{1-s_{1}}$ to cancel out $\left|k_{1}+k_{2}\right|^{1-s_{1}}$ in the numerator of (4.15).

(3.2.1) $\left|\lambda_{1}+k_{1}^{2}\right|^{1 / 2} \geq\left|k_{1}\right|$

Estimate (4.15) by

$$
\begin{gathered}
\sum_{k_{1}, k_{2}} \int d \lambda_{1} d \lambda_{2} d \lambda \\
\frac{\bar{c}\left(k_{1}, \lambda_{1}\right) c\left(k_{2}, \lambda_{2}\right) \overline{\bar{c}}\left(-\frac{k_{1}+k_{2}}{2}, \lambda-k_{1}-k_{2}\right) d\left(\frac{k_{1}+k_{2}}{2}, \lambda\right)}{\left|\left(\lambda_{1}+\lambda_{2}\right) \pm\left(k_{1}+k_{2}\right)\right|\left|\lambda_{1}+k_{1}^{2}\right|^{\frac{1}{2}}\left|\lambda_{2}-k_{2}^{2}\right|^{1 / 2}\left|\lambda-k_{1}-k_{2}+\left(\frac{k_{1}+k_{2}}{2}\right)^{2}\right|^{1 / 2}\left|\lambda+\left(\frac{k_{1}+k_{2}}{2}\right)^{2}\right|^{1 / 2}}
\end{gathered}
$$

and hence, by $\int F_{2} G_{1} \leq\left\|F_{2}\right\|_{2}\left\|G_{1}\right\|_{2}$, where

$$
\begin{gathered}
F_{2}=\sum_{k_{1}, k_{2}} e^{i\left(k_{1}+k_{2}\right)\left(\frac{x}{2}+t\right)}\left\{\int d \lambda_{1} d \lambda_{2} \frac{\bar{c}\left(k_{1}, \lambda_{1}\right) \bar{c}\left(k_{2}, \lambda_{2}\right)}{\left|\left(\lambda_{1}+\lambda_{2}\right) \pm\left(k_{1}+k_{2}\right)\right|\left|\lambda_{1}+k_{1}^{2}\right|^{\frac{1}{2}}\left|\lambda_{2}-k_{2}^{2}\right|^{1 / 2}}\right\} . \\
\left\{\int d \lambda_{3} e^{i \lambda_{3} t} \frac{\overline{\bar{c}}\left(-\frac{k_{1}+k_{2}}{2}, \lambda_{3}\right)}{\left|\lambda_{3}+\left(\frac{k_{1}+k_{2}}{2}\right)^{2}\right|^{1 / 2}}\right\} \\
G_{1}=\sum_{k} \int d \lambda \frac{d(k, \lambda)}{\left|\lambda+k^{2}\right|^{1 / 2}} e^{-i(k x+\lambda t)} .
\end{gathered}
$$

To bound $\left\|F_{2}\right\|_{2}$, consider $\{\omega(\ell)\}$ with $\sum \omega(\ell)^{2} \leq 1$ and the expression

$$
\sum_{k_{1} k_{2}} \int d \lambda_{1} d \lambda_{2} \frac{\omega\left(k_{1}+k_{2}\right)}{\left|\lambda_{1}+k_{1}^{2}\right|^{\frac{31}{2}}\left|\lambda_{2}-k_{2}^{2}\right|^{1 / 2}\left|\left(\lambda_{1}+\lambda_{2}\right) \pm\left(k_{1}+k_{2}\right)\right|} \bar{c}\left(k_{1}, \lambda_{1}\right) \bar{c}\left(k_{2}, \lambda_{2}\right) .
$$

When applying Shur's criterion on the $\left(\left(k_{1}, \lambda_{1}\right),\left(k_{2}, \lambda_{2}\right)\right)$-matrix in $(4.21)$, the $\left(\lambda_{1}, k_{1}\right)$ - summation yields by (4.9) and assumption on $\omega$

$$
\sum_{k_{1}} \frac{\omega\left(k_{1}+k_{2}\right)}{1+\left|k_{1}^{2} \pm\left(k_{1}+k_{2}\right)-\lambda_{2}\right|^{\frac{1}{2}-}} \leq\left(\sum_{k_{1}} \frac{1}{1+\left|k_{1}^{2} \pm\left(k_{1}+k_{2}\right)-\lambda_{2}\right|^{s_{1}-}}\right)^{1 / 2}<c
$$

by (4.11) since $s_{1}>\frac{1}{2}$.

(3.2.2) $\left|\lambda_{2}-k_{2}^{2}\right|^{1 / 2} \geq\left|k_{1}\right|$ 
idem as (3.2.1).

(3.2.3) $\left|\left(\lambda_{1}+\lambda_{2}\right) \pm\left(k_{1}+k_{2}\right)\right|^{1 / 2} \gtrsim\left|k_{1}\right|$

We then estimate (4.15) by

$$
\begin{gathered}
\sum_{k_{1}, k_{2}} \int d \lambda_{1} d \lambda_{2} d \lambda \\
\frac{\bar{c}\left(k_{1}, \lambda_{1}\right) \bar{c}\left(k_{2}, \lambda_{2}\right) \overline{\bar{c}}\left(-\frac{k_{1}+k_{2}}{2}, \lambda-k_{1}-k_{2}\right) d\left(\frac{k_{1}+k_{2}}{2}, \lambda\right)}{\left|\left(\lambda_{1}+\lambda_{2}\right) \pm\left(k_{1}+k_{2}\right)\right|^{\frac{1+k_{1}}{2}}\left|\lambda_{1}+k_{1}^{2}\right|^{1 / 2}\left|\lambda_{2}-k_{2}^{2}\right|^{1 / 2}\left|\lambda+\left(\frac{k_{1}+k_{2}}{2}\right)^{2}\right|^{1 / 2}}
\end{gathered}
$$

and let

$$
\begin{aligned}
F_{3}=\sum_{k_{1}, k_{2}} e^{i\left(k_{1}+k_{2}\right)\left(\frac{\Gamma}{2}+t\right)}\left\{\int d \lambda_{1} d \lambda_{2} \frac{\bar{c}\left(k_{1}, \lambda_{1}\right) \bar{c}\left(k_{2}, \lambda_{2}\right)}{\left|\left(\lambda_{1}+\lambda_{2}\right) \pm\left(k_{1}+k_{2}\right)\right|^{\frac{1+\alpha_{1}}{2}}\left|\lambda_{1}+k_{1}^{2}\right|^{1 / 2}\left|\lambda_{2}-k_{2}^{2}\right|^{1 / 2}}\right\} . \\
\\
\left\{\int d \lambda_{3} e^{i \lambda_{3} t} \overline{\bar{c}}\left(-\frac{k_{1}+k_{2}}{2}, \lambda_{3}\right)\right\} .
\end{aligned}
$$

Thus we bound (4.22) by $\int F_{3} \cdot G_{1} \leq\left\|F_{3}\right\|_{2} \cdot\left\|G_{1}\right\|_{2}$ and the estimate on $\left\|F_{3}\right\|_{2}$ amount to apply Shur's criterion on the matrix

$$
\left\{\frac{\omega\left(k_{1}+k_{2}\right)}{\left|\left(\lambda_{1}+\lambda_{2}\right) \pm\left(k_{1}+k_{2}\right)\right|^{\frac{1+\sigma_{1}}{2}}\left|\lambda_{1}+k_{1}^{2}\right|^{1 / 2}\left|\lambda_{2}-k_{2}^{2}\right|^{1 / 2}}\right\}_{\substack{\left(k_{1}, \lambda_{1}\right) \\\left(k_{2}, \lambda_{2}\right)}}
$$

where $\sum_{\ell} \omega(\ell)^{2} \leq 1$. Performing the $\lambda_{1}$-integration in (4.24), one gets from (4.9) $\left(\frac{1}{2}<s_{1}<1\right)$ again

$$
\sum_{k_{1}} \frac{\omega\left(k_{1}+k_{2}\right)}{\left|k_{1}^{2} \pm\left(k_{1}+k_{2}\right)-\lambda_{2}\right|^{\frac{s_{1}}{2}}}<\left(\sum_{k_{1}} \frac{1}{\left|k_{1}^{2} \pm\left(k_{1}+k_{2}\right)+\lambda_{2}\right|^{s_{1}}}\right)^{1 / 2}<c
$$

Next we have to bound for fixed $k$

$$
\begin{gathered}
\sum_{k=k_{1}+k_{2}+k_{3}} \int d \lambda_{1} d \lambda_{2} d \lambda \\
\frac{\left|k^{s_{1}}\right| k_{1}+k_{2} \mid c\left(k_{1}, \lambda_{1}\right) c\left(k_{2}, \lambda_{2}\right) c\left(k_{3}, \lambda-k_{1}-k_{2}\right) d(k, \lambda)}{\left|\left(\lambda_{1}+\lambda_{2}\right) \pm\left(k_{1}+k_{2}\right)\right|\left|\lambda_{1}+k_{1}^{2}\right|^{1 / 2}\left|\lambda_{2}-k_{2}^{2}\right|^{1 / 2}\left|\lambda-k_{1}-k_{2}+k_{3}^{2}\right|^{1 / 2}\left|\lambda+k^{2}\right|^{1 / 2}}
\end{gathered}
$$

when verifying $\|\{\overline{\bar{c}}(k, \lambda)\}\|_{\ell_{k}^{\infty} L_{\lambda}^{2}}$. In the cases (1), (2), there is a gain of a $|k|^{\varepsilon}$-factor for some (numerical) $\varepsilon>0$, as pointed out. Thus if $s_{1}<s+\varepsilon$, the $\|\overline{\bar{c}}\|_{\ell_{k}^{\infty} L_{\lambda}^{2}}\left(\leq\|\overline{\bar{c}}\|_{\ell_{k}^{2} L_{\lambda}^{2}}\right)$ bound is already obtained, except in the case $k_{1}+k_{2}+2 k_{3}=0(1)$. Assume $k_{1}+k_{2}+2 k_{3}=0$, say. We have to estimate (cf. (4.15)) 


$$
\begin{gathered}
\sum_{k_{1}+k_{2}=\ell} \int d \lambda_{1} d \lambda_{2} d \lambda \\
\frac{|\ell|^{1-s} \bar{c}\left(k_{1}, \lambda_{1}\right) c\left(k_{2}, \lambda_{2}\right) \overline{\bar{c}}\left(-\frac{\ell}{2}, \lambda-\ell\right) d(\lambda)}{\left|\left(\lambda_{1}+\lambda_{2}\right) \pm \ell\right| \lambda_{1}+\left.k_{1}^{2}\right|^{1 / 2}\left|\lambda_{2}-k_{2}^{2}\right|^{1 / 2}\left|\lambda-\ell+\left(\frac{\ell}{2}\right)^{2}\right|^{1 / 2}\left|\lambda+\left(\frac{\ell}{2}\right)^{2}\right|^{1 / 2}}
\end{gathered}
$$

for fixed $\ell$. Here $\int d(\lambda)^{2} d \lambda \leq 1$. Applying Hölder's inequality when performing the $\lambda$-integration yields for $(4.26)$

$$
\sum_{k_{1}} \int d \lambda_{1} d \lambda_{2} \frac{|\ell|^{1-s} \bar{c}\left(k_{1}, \lambda_{1}\right) c\left(\ell-k_{2}, \lambda_{2}\right)}{\left|\left(\lambda_{1}+\lambda_{2}\right) \pm \ell\right|\left|\lambda_{1}+k_{1}^{2}\right|^{1 / 2}\left|\lambda_{2}-\left(\ell-k_{1}\right)^{2}\right|^{1 / 2}}
$$

(3.1) $\left|k_{1}\right| \sim\left|\ell-k_{1}\right|, \ell-2 k_{1} \neq 0(1)$

(4.27) yields

$$
\sum_{k_{1}} \int d \lambda_{1} d \lambda_{2} \frac{|\ell|^{1-2 s} \bar{c}\left(k_{1}, \lambda_{1}\right) \bar{c}\left(\ell-k_{1}, \lambda_{2}\right)}{\left|\left(\lambda_{1}+\lambda_{2}\right) \pm \ell\right|\left|\lambda_{1}+k_{1}^{2}\right|^{1 / 2}\left|\lambda_{2}-\left(\ell-k_{1}\right)^{2}\right|^{1 / 2}}
$$

Using $(4.2)^{2(1-2 s)}$ gives then a bound by one of the expressions

$$
\begin{gathered}
\sum_{k_{1}} \int d \lambda_{1} d \lambda_{2} \frac{\bar{c}\left(k_{1}, \lambda_{1}\right) \bar{c}\left(\ell-k_{1}, \lambda_{2}\right)}{\left|\left(\lambda_{1}+\lambda_{2}\right) \pm \ell\right|\left|\lambda_{1}+k_{1}^{2}\right|^{2 s-\frac{1}{2}}\left|\lambda_{2}-\left(\ell-k_{1}\right)^{2}\right|^{1 / 2}} \\
\sum_{k_{1}} \int d \lambda_{1} d \lambda_{2} \frac{\bar{c}\left(k_{1}, \lambda_{1}\right) \bar{c}\left(\ell-k_{1}, \lambda_{2}\right)}{\left|\left(\lambda_{1}+\lambda_{2}\right) \pm \ell\right|\left|\lambda_{1}+k_{1}^{2}\right|^{\frac{1}{2}}\left|\lambda_{2}-\left(\ell-k_{1}\right)^{2}\right|^{2 s-\frac{1}{2}}} \\
\sum_{k_{1}} \int d \lambda_{1} d \lambda_{2} \frac{\bar{c}\left(k_{1}, \lambda_{1}\right) \bar{c}\left(\ell-k_{1}, \lambda_{2}\right)}{\left|\left(\lambda_{1}+\lambda_{2}\right) \pm \ell\right|^{2 s}\left|\lambda_{1}+k_{1}^{2}\right|^{\frac{1}{2}}\left|\lambda_{2}-\left(\ell-k_{1}\right)^{2}\right|^{\frac{1}{2}}}
\end{gathered}
$$

First integrate in $\lambda_{1}, \lambda_{2}$ considering the denominators in (4.29), (4.30) as $\left(\lambda_{1}, \lambda_{2}\right)$-matrix element. By Shur, one bounds by $\left(\int \bar{c}\left(k_{1}, \lambda_{1}\right)^{2} d \lambda_{1}\right)^{1 / 2}\left(\int \bar{c}\left(\ell-k_{1}, \lambda_{2}\right)^{2} d \lambda_{2}\right)^{1 / 2}$. Here we assume $s>\frac{1}{4}$. The resulting $k_{1}$ summation is bounded by $\|\bar{c}\|_{\ell_{k}^{2} L_{\lambda}^{2}}^{2}$ from Hölder's inequality.

(3.3) $\ell-2 k_{1}=0(1)$

Let $k_{1}=\frac{\ell}{2}$, say. Then (4.27) becomes

$$
\int d \lambda_{1} d \lambda_{2} \frac{|\ell|^{1-2 s_{1}} \overline{\bar{c}}\left(\frac{\ell}{2}, \lambda_{1}\right) \overline{\bar{c}}\left(\frac{\ell}{2}, \lambda_{2}\right)}{\left|\left(\lambda_{1}+\lambda_{2}\right) \pm \ell\right|\left|\lambda_{1}+\left(\frac{\ell}{2}\right)^{2}\right|^{1 / 2}\left|\lambda_{2}-\left(\frac{\ell}{2}\right)^{2}\right|^{1 / 2}} \leq\|\overline{\bar{c}}\|_{\ell_{k}^{\infty} L_{\lambda}^{2}}^{2}
$$

estimating the $\left(\lambda_{1}, \lambda_{2}\right)$-integration as above.

(3.2) $\left|k_{1}\right| \gg\left|\ell-k_{1}\right|$ 
Then (4.2) $\geq\left|k_{1}\right|$ and we consider (4.2) $)^{1-s}$ to cancel out $|\ell|^{1-s}$ in (4.27). In (4.29), one gets the denominators $\left|\left(\lambda_{1}+\lambda_{2}\right) \pm \ell\right|\left|\lambda_{1}+k_{1}^{2}\right|^{s / 2}\left|\lambda_{2}-\left(\ell-k_{1}\right)^{2}\right|^{1 / 2}$ or $\left|\left(\lambda_{1}+\lambda_{2}\right) \pm \ell\right|\left|\lambda_{1}+k_{1}^{2}\right|^{1 / 2}\left|\lambda_{2}-\left(\ell-k_{1}^{2}\right)\right|^{s / 2}$ and in (4.30) the denominator $\left|\left(\lambda_{1}+\lambda_{2}\right) \pm \ell\right|^{\frac{1}{2}+\frac{2}{2}}\left|\lambda_{1}+k_{1}^{2}\right|^{1 / 2}\left|\lambda_{2}-\left(\ell-k_{1}\right)^{2}\right|^{1 / 2}$. The $\left(\lambda_{1}, \lambda_{2}\right)$-integration may thus again be estimated by Shur's Lemma.

\section{Estimation of (1.20)}

The same comments apply as for (1.18). Again the estimates in (1.21) are clearly not tied.

\section{Estimation of (1.22) and (1.23)}

Recall that in both sums the terms $k^{\prime}=0,-2 k_{3} \pm 1$ (with matching sign) are deleted.

Consider first (1.23). The contribution to the \|\|$_{\ell_{k}^{2} L_{\lambda}^{2}}-$ norm is bounded by

$$
\sum_{\substack{k^{\prime}, k_{3} \\ k^{\prime} \neq 0_{0},-2 k_{3} \pm 1}} \int d \lambda \frac{\left|k_{3}+k^{1}\right|^{s}\left|c_{k^{3}}\right| c\left(k_{3}, \lambda \pm k^{1}\right) d\left(k_{3}+k^{1}, \lambda\right)}{\left|\lambda \pm k^{1}+k_{3}^{2}\right|^{1 / 2}\left|\lambda+\left(k_{3}+k^{1}\right)^{2}\right|^{1 / 2}}
$$

where

$$
\sum_{k} \int d \lambda d(k, \lambda)^{2} \leq 1, d(k, \lambda) \geq 0 \text { and }\left|c_{k^{1}}\right|<\left|k^{1}\right|^{\sigma} .
$$

Observe that for the denominators

$$
\max \left\{\left|\lambda \pm k^{1}+k_{3}^{2}\right|,\left|\lambda+\left(k_{3}+k^{\prime}\right)^{2}\right|\right\} \geq\left|k^{\prime}\right|\left|2 k_{3}+k^{\prime} \mp 1\right|
$$

where from assumption on the summation in (5.1) non of the factors in (5.3) vanishes

(1) $\left|k_{3}\right| \geq\left|k^{1}\right|$

Estimate (5.1) by

$$
\sum_{k_{3}, k^{\prime} \neq 0,-2 k_{3} \pm 1} \int d \lambda \frac{\left|k^{\prime}\right|^{\sigma} \bar{c}\left(k_{3}, \lambda \pm k^{1}\right) d\left(k_{3}+k^{1}, \lambda\right)}{\left|\lambda \pm k^{1}+k_{3}^{2}\right|^{1 / 2}\left|\lambda+\left(k_{3}+k^{1}\right)^{2}\right|^{\frac{1}{2}}}
$$

Since (5.3) is at least $\left|k^{1}\right|$, one of the denominatots is at least $\left|k^{1}\right|^{\frac{1}{2}}$ and we bound (5.4) writing

$$
\begin{aligned}
& \sum_{k_{3}, k^{1}} \int d \lambda \frac{\bar{c}\left(k_{3}, \lambda\right) d\left(k_{3}+k^{1}, \lambda \mp k^{1}\right)}{\left|\lambda+k_{3}^{2}\right|^{1 / 2-\sigma}\left|\lambda \mp k^{1}+\left(k_{3}+k^{1}\right)^{2}\right|^{1 / 2-\sigma}} \\
\leq & \|\bar{c}\|_{\ell_{k}^{2} L_{\lambda}^{2}}\left[\sum_{k_{3}} \int d \lambda \frac{1}{\left|\lambda+k_{3}^{2}\right|^{1-2 \sigma}}\left(\sum_{k^{1}} \frac{d\left(k_{3}+k^{1}, \lambda \mp k^{1}\right)}{\left|\lambda \mp k^{1}+\left(k_{3}+k^{1}\right)^{2}\right|^{\frac{1}{2}-\sigma}}\right)^{2}\right]^{1 / 2} \\
\leq & \|\bar{c}\|\left[\sum_{k_{3}} \int d \lambda \frac{1}{\left|\lambda+k_{3}^{2}\right|^{1-2 \sigma}} \sum_{k^{1}} d\left(k_{3}+k^{1}, \lambda \mp k^{1}\right)^{2}\right]^{1 / 3} \\
\leq & \|\bar{c}\|\left[\sum_{k_{3}, k^{1}} \int d \lambda \frac{d\left(k^{1}, \lambda \mp k^{1} \pm k_{3}\right)^{2}}{\left|\lambda+k_{3}^{2}\right|^{1-2 \sigma}}\right]^{1 / 2}
\end{aligned}
$$




$$
\begin{aligned}
& \leq\|\bar{c}\|\left[\sum_{k_{3}, k^{1}} \int d \lambda \frac{d\left(k^{1}, \lambda\right)^{2}}{\left|\lambda \pm k^{1} \mp k_{3}+k_{3}^{2}\right|^{1-2 \sigma}}\right]^{1 / 2} \\
& \leq\|\bar{c}\|\|\{d\}\|=\|\bar{c}\|
\end{aligned}
$$

provided $1-2 \sigma>\frac{1}{2}$. We used here Hölder's inequality, (4.11) and variable changes.

(2) $\left|k^{1}\right| \gg\left|k_{3}\right|$

(5.1) becomes

$$
\sum_{k_{3}, k^{1}} \int d \lambda \frac{\left|k^{1}\right|^{s+\sigma} c\left(k_{3}, \lambda \pm k^{1}\right) d\left(k_{3}+k^{1}, \lambda\right)}{\left|\lambda \pm k^{1}+k_{3}^{2}\right|^{1 / 2}\left|\lambda+\left(k_{3}+k^{1}\right)^{2}\right|^{1 / 2}}
$$

where now, by (5.3), the maximum of the denominators is at least $\left|k^{1}\right|$. The preceding calculation is clearly conclusive provided $s+\sigma$

$$
s+\sigma<\frac{1}{2} .
$$

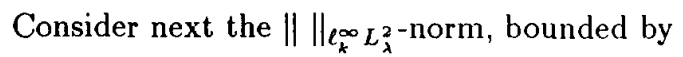

$$
\sup _{k}|k|^{* 1} \sum_{k^{1} \neq 0,2 k \mp 1} \int d \lambda \frac{\left|k^{1}\right|^{\sigma} c\left(k-k^{1}, \lambda \pm k^{1}\right) d(\lambda)}{\left|\lambda \pm k^{1}+\left(k-k^{1}\right)^{2}\right|^{1 / 2}\left|\lambda+k^{2}\right|^{1 / 2}}
$$

where $\int d(\lambda)^{2} \leq 1, d(\lambda) \geq 0$.

The argument in case (1) above permits to bound (5.8) also, since the $|k|^{s}$-factor may have been replaced by $|k|^{s_{1}}$, provided $s_{1}-s+\sigma<\frac{1}{4}$. Assume thus case (2), i.e. $\left|k^{1}\right| \gg\left|k-k^{1}\right|$. Since the denorminator in (5.8) is at least $|k|$, we get applying Hölder's inequality

$$
|k|^{s_{1}+\sigma-1} \sum_{k^{2},\left|k^{1}\right| \sim|k|}\left\|\bar{c}\left(k-k^{1}, \cdot\right)\right\|_{L_{\lambda}^{2}}\left(1+\left|k-k^{1}\right|\right)^{-s} \leq|k|^{-\frac{1}{2}-s+s_{1}+\sigma}\|\bar{c}\|_{\ell_{k}^{2} L_{\lambda}^{2}} .
$$

This is certainly conclusive if $s_{1}-s+\sigma<\frac{1}{4}$.

In the (1.23)-analysis, the factor $\frac{1}{\left|\lambda+k^{2}\right|^{\frac{1}{2}}}$ may be replaced by $\frac{1}{\left|\lambda+k^{2}\right|^{\frac{1}{2}}-}$, so that the preceding also yields the estimate on (1.22).

This completes the estimates on (1.18)-(1.23), hence the (1.17)-norm of (1.11)-(1.16) and therefore (0.8) and the solution $u$ of the modified equation (cf. (0.9))

$$
\left\{\begin{array}{c}
i u_{t}+u_{x x}-L u=u_{0} \square^{-1}\left(\left|u_{0}\right|^{2}\right)_{x x}+\left[u_{0} W(t)(a, b)-L u_{0}\right] \\
u(x, 0)=\varphi(x)
\end{array}\right.
$$

\section{Application of the contraction principle}

Let $T$ be the transformation $u_{0} \mapsto u$ defined by (5.10), i.e.

$$
i u_{t}+u_{x x}-L u=u_{0} \square^{-1}\left(\left|u_{0}\right|^{2}\right)_{x x}+u_{0} W(t)(a, b)-L u_{0}, u(x, 0)=\varphi(x) .
$$

Here $\varphi, a, b$ fulfil the hypothesis of Theorem 1, i.e. 


$$
\varphi \in H^{s}(\mathrm{~T}), \sup _{k}|k|^{s_{2}}|\widehat{\varphi}(k)|<\infty \text { and } \sup _{k}\left(|k|^{-\sigma}|\widehat{a}(k)|,|k|^{-\sigma-1}|\widehat{b}(k)|\right)<\infty .
$$

It follows from the preceding that for suitable values of $0<s<\frac{1}{2}<s_{1}<1, \sigma>0$, the transformation $T$ maps the ball

$$
\|\bar{c}\|_{\ell_{k}^{2} L_{\lambda}^{2}} \leq 1, \quad\|\bar{c}\|_{\ell_{k}^{\infty} L_{\lambda}^{2}} \leq 1
$$

denoting

$$
\bar{c}(k, \lambda)=\left(1+|k|^{s}\right)\left(1+\left|\lambda+k^{2}\right|^{1 / 2}\right)|\widehat{u}(k, \lambda)| \text { and } \overline{\bar{c}}(k, \lambda)=\left(1+|k|^{s_{1}}\right)\left(1+\left|\lambda+k^{2}\right|^{1 / 2}\right)|\widehat{u}(k, \lambda)|
$$

in a bounded set in the same space. From the systematic gains appearing in the above calculations, there is a saving on the denominator factors $\left|\lambda_{i} \pm k_{i}^{2}\right|^{1 / 2}$, allowing to obtain estimates in terms of $\|u\|^{1-\delta}\|u\|_{2}^{\delta}$ rather than $\|u\|$, for some $\delta>0$. This feature permits to verify a contraction property of $T$ for the previous norm, provided one restricts to a sufficiently small time interval (these considerations are similar to those in $\left[\mathrm{B}_{1,2}\right]$ and we don't elaborate them here). Hence the fixpoint theorem applies and yields a solution to (0.9) hence (0.8). This proves Theorem 1 .

It remains to verify the properties of $n$, defined by equation (II)

$$
\left\{\begin{array}{l}
n_{t t}-n_{x x}=\Delta|u|^{2} \\
n(x, 0)=a, \partial_{t} n(x, 0)=b .
\end{array}\right.
$$

We also consider the auxiliary field $V=V(x, t)$ defined by

$$
\left\{\begin{array}{l}
V_{x}=-n_{t} \\
V_{t}=-n_{x}-|u|_{x}^{2}
\end{array} \quad \int V(x, t) d x=0 .\right.
$$

We then have a hamiltonian setup

$$
\left\{\begin{array}{l}
i u_{t}=-u_{x x}+n u \\
V_{t}=-n_{x}-|u|_{x}^{2} \\
n_{t}=-V_{x}
\end{array}\right.
$$

with Hamiltonian

$$
H=\int_{\mathbf{T}}\left[\left|u_{x}\right|^{2}+\frac{1}{2}\left(n^{2}+V^{2}\right)+n|u|^{2}\right] d x
$$

with $\left(\operatorname{Re} u, \operatorname{Im} u\right.$ ) and $(\tilde{n}, \tilde{V})$ as pairs of conjugate variables, where $\widetilde{n}=2^{-1 / 2} n, \widetilde{V}=2^{-1 / 2} D_{x}^{-1} V$ (see [L-R-S]). For initial data $(\varphi, a, b)$ in $\left(H^{1}, L^{2}, H^{-1}\right),(6.7)$ gives thus a priori bounds on $\|u(\cdot, t)\|_{H^{1}},\|n(\cdot, t)\|_{L^{2}}$, $\left\|\partial_{t} n(\cdot, t)\right\|_{H^{-1}}$. Theorem 2 follows from this fact and Theorem 1.

\section{Estimation of $n$ and $V$}

Considering the weaker assumptions on $\varphi, a, b$ from Theorem 1, one may bound $\|n(\cdot, t)\|_{2},\|V(\cdot, t)\|_{2}$ from (0.5), (1.5) and the estimates obtained for $u$ (considering the local problem).

Considering the contribution of the first term $\square^{-1}\left(|u|^{2}\right)_{x x}$ in $(0.5)$, thus

$$
\left\|\square^{-1}\left(|u|^{2}\right)_{x x}(t)\right\|_{2}+\left\|\partial_{t} \square^{-1}\left(|u|^{2}\right)_{x}(t)\right\|_{2}
$$

formula (1.5) yields the estimate. 


$$
\begin{gathered}
\sum_{k_{1}, k_{2}} \int d \lambda_{1} d \lambda_{2}|k| \frac{c\left(k_{1}, \lambda_{1}\right) c\left(k_{2}, \lambda_{2}\right) d(k)}{|\lambda \pm k|\left|\lambda_{1}+k_{1}^{2}\right|^{1 / 2}\left|\lambda_{2}-k_{2}^{2}\right|^{1 / 2}}+ \\
\sum_{k_{1}, k_{2}} \int d \lambda_{1} d \lambda_{2}|k|(|k|+|\lambda|) \frac{c\left(k_{1}, \lambda_{1}\right) c\left(k_{2}, \lambda_{2}\right) d(k)}{|\lambda+k||\lambda-k|\left|\lambda_{1}+k_{1}^{2}\right|^{1 / 2}\left|\lambda_{2}-k_{2}^{2}\right|^{1 / 2}} \underset{\substack{\left.\lambda=\lambda_{1}+\lambda_{2}\right) \\
\left(k=k_{1}+k_{2}\right)}}{ }
\end{gathered}
$$

where $\sum_{k} d(k)^{2} \leq 1$

One of the factors $\lambda+k$ or $\lambda-k$ takes care of $|k|+|\lambda|$. Observe that

$$
\max \left(|\lambda \pm k|^{1 / 2},\left|\lambda_{1}-k_{1}^{2}\right|^{1 / 2},\left|\lambda_{2}+k_{2}^{2}\right|^{1 / 2}\right) \geq|k|^{1 / 2}\left|k_{1}-k_{2} \pm 1\right|^{1 / 2} .
$$

Assume $\left|k_{1}\right| \geq\left|k_{2}\right|$. A straightforward analysis considering the different cases $\left|k_{1}\right| \gg\left|k_{2}\right|,\left|k_{1}\right| \sim\left|k_{2}\right|$, $k_{1}-k_{2}=0(1)$ in the spirit of the previous arguments shows that (7.2) is bounded. In the case $k_{1}-k_{2}=0(1)$, also the second part of the norm (1.17) $\|u\|$ is used again. We skip details.

It follows thus from (0.5) that up to the $W(t)(a, b)$ (resp. $\left.\partial_{t} D_{x}^{-1} W(t)(a, b)\right)$ term, $\|n(\cdot, t)\|_{2},\|V(\cdot, t)\|_{2}$ are bounded.

In particular $n(\cdot, t)$ and $\partial_{t} n(\cdot, t)$ satisfy the assumption on $a($ resp. $b$ ) of Theorem 1.

\section{Invariant measures}

Letting $H$ be the Hamiltonian (6.7), the formal Gibbs measure

$$
e^{-\beta H} \chi_{\left\{\int|u|^{2} d x \leq B\right\}} \prod_{x}\left[d^{2} u(x) d \tilde{n}(x) d \tilde{V}(x)\right]
$$

with $\tilde{n}=2^{-1 / 2} n, \tilde{V}=2^{-1 / 2} D_{x}^{-1} V$ is formally invariant under the flow (6.6).

Observe that $H$ may be rewritten as

$$
H=\int_{\mathbf{T}}\left[\left|u_{x}\right|^{2}-\frac{1}{2}|u|^{4}+\frac{1}{2}\left(n+|u|^{2}\right)^{2}+\frac{1}{2} V^{2}\right]
$$

and denoting $\tilde{\tilde{n}}=2^{-1 / 2}\left(n+|u|^{2}\right)$, (8.1) gives

$$
\begin{gathered}
\left\{e^{\frac{1}{2} \int|u|^{4}} \chi_{\left\{\|u\|_{2}^{2} \leq B\right\}} e^{-\int\left|u_{x}\right|^{2}}\left[\prod_{x} d^{2} u(x)\right]\right\}\left\{e^{-\int(\tilde{\tilde{n}})^{2}}\left[\prod_{x} d \tilde{\tilde{n}}(x)\right]\right\} \\
\left\{e^{-\int\left(\widetilde{V}_{x}\right)^{2}}\left[\prod_{x} d \tilde{V}(x)\right]\right\}
\end{gathered}
$$

(the first part is the invariant measure for the NLSE) so that after normalization one except as generic elements $(u, \tilde{\tilde{n}}, \widetilde{V})$ in its support random Fourier series of the form

$$
\begin{gathered}
u=\sum \frac{1}{k} G_{k}(\omega) e^{i k x} \\
\widetilde{\widetilde{n}}=\sum\left(g_{k}^{1}(\omega) \cos k x+g_{k}^{2}(\omega) \sin k x\right)
\end{gathered}
$$




$$
\tilde{V}=\sum \frac{1}{k}\left(g_{k}^{3}(\omega) \cos k x+g_{k}^{4}(\omega) \sin k x\right)
$$

where $\left\{g_{k}^{i}\right\}(i=1,2,3,4)$ are independent Gaussians and $\left\{G_{k}\right\}$ independent complex valued Gaussians. Thus (8.4) clearly satisfies almost surely the hypothesis on the data $\varphi$ in Theorem 1, i.e. $\left(s<\frac{1}{2}<s_{1}<1\right)$

Choosing $\sigma>0$, one has for (8.5) almost surely

$$
\varphi \in H^{s}(\mathrm{~T}) \text { and } \sup |k|^{s_{1}}|\hat{\varphi}(k)|<\infty .
$$

$$
\sup _{k}|k|^{-\sigma}|\widehat{\tilde{\tilde{n}}}(k)|<\infty
$$

implying, since $\tilde{\tilde{n}}=2^{-1 / 2}\left(n+|u|^{2}\right)$, also

$$
\sup _{k}|k|^{-\sigma}|\widehat{n}(k)|<\infty
$$

(8.6) yields

$$
V_{x}=\sum k\left(g_{k}^{3}(\omega) \cos k x+g_{k}^{4} \sin k x\right)
$$

hence, from the equation $n_{t}=-V_{x}$

$$
\sup _{k}|k|^{-\sigma-1}\left|\widehat{n}_{t}(k)\right|<\infty .
$$

Thus (8.9), (8.11) were the assumptions made on the data $a, b$ in Theorem 1 .

To prove Theorem 3, i.e. the fact that the Gibbs measure is supported by an invariant set on which the Zakharov system is globally wellposed, we repeat the steps in $\left[\mathrm{Bo}_{3}\right]$ for NLS equations. Thus first, one considers the finite dimensional model, obtained by considering only frequences $|k| \leq N$ and the modified system

$$
\begin{cases}i u_{t}^{N}=-u_{x x}^{N}+P_{N}\left(n^{N} u^{N}\right) & u^{N}(x, 0)=\varphi^{N}(x) \\ n_{t}^{N}=-V_{x}^{N} & n^{N}(x, 0)=a^{N}(x) \\ V_{t}^{N}=-n_{x}^{N}+P_{N}\left(\left|u^{N}\right|_{x}^{2}\right) & V^{N}(x, 0)=D_{x}^{-1} b^{N}(x)\end{cases}
$$

where $u^{N}, n^{N}, V^{N}$ have Fourier series with respect to the $x$-variable restricted to $|k| \leq N$ and $P_{N}$ is the usual Dirichlet projection. The proof of Theorem 1 yields local wellposedness of (8.12) with bounds independent of $N$. In particular, the size of the time $T$ is controlled by the data (in a polynomial way) and will be at least $\tau>0$ on a subset of the statistical ensemble which (normalized) Gibbs measure is $>1-e^{-\frac{1}{r^{c}}}$ (fixing any small $\tau$ ). The phase space is obtained by considering the Fourier transforms of $u, n, V$ as canonical variables (rather than the functions themself) and the introductory remarks in the beginning of this section are rigorous because finite dimensional. In particular, one has invariant measures and the reasoning in [Bo $\left.{ }_{3}\right]$ for NLS $i u_{t}+u_{x x}+u|u|^{p-2}=0$ with $p>4$ may be repeated here. Thus the invariant measure is used as a conservation law when braking up a given time interval in small subintervals for which Theorem 1 applies. This reasoning yields for any $\delta>0$ a subset of the phase space of complementary Gibbs measure at most $\delta$ and on which (8.12) is globally wellposed (with bound independent of $N$ ) with estimates on $u^{N}(t, \cdot), n^{N}(t, \cdot)$ and $V^{N}(t, \cdot)$ of the form

$$
\left(\log \frac{|t|+1}{\delta}\right)^{1 / 2} \geq\left\|u^{N}(t)\right\| \|_{H} \cdot+\sup _{|k| \leq N}\left(|k|^{s_{1}}\left|\widehat{u^{N}(t)}(k)\right|+|k|^{-\sigma}\left(\left|n^{N}(t)(k)\right|+\left|\widehat{V^{N}(t)}(k)\right|\right)\right.
$$

for any choosen $0<\sigma, 0<s<\frac{1}{2}<s_{1}<1$ such that Theorem 1 is applicable.

Once the finite models analyzed (with uniform estimates in $N$ ), Theorem 3 for the original system is then ( $€$ asily) completed as in $\left[\mathrm{Bo}_{3}\right]$. 


\section{References}

$\left[\mathrm{B}_{1,2}\right]$ J. BOURGAIN, Fourier restriction phenomena for certain lattice subsets and applications to nonlinear evolution equations, GAFA, 3 (1993), Part I, 107-156, Part II, 209-262.

$\left[\mathrm{B}_{3}\right]$ J. BOURGAIN, Periodic nonlinear Schrödinger equations and invariant measures, preprint IHES, Summer 93 .

[L-R-S] J. LEBOWITZ, H. ROSE, E. SPEER, Statistical mechanics of the nonlinear Schrödinger equation, J. of Stat. Phys., Vol. 5, $\mathrm{N}^{0} 3 / 4(1988), 657-687$.

[O-T] T. OZAWA, Y. TSUTSUMI, Existence and smoothing effect of solutions for the Zakharov equation, Diff. and Int. Eqs., 5 (1992), 721-745. 
